\title{
NORM ESTIMATES FOR RADIALLY SYMMETRIC SOLUTIONS OF SEMILINEAR ELLIPTIC EQUATIONS
}

\author{
RYUJI KAJIKIYA
}

\begin{abstract}
The semilinear elliptic equation $\Delta u+f(u)=0$ in $R^{n}$ with the condition $\lim _{|x| \rightarrow \infty} u(x)=0$ is studied, where $n \geq 2$ and $f(u)$ has a superlinear and subcritical growth at $u= \pm \infty$. For example, the functions $f(u)=|u|^{p-1} u-u(1<p<\infty$ if $n=2,1<p<(n+2) /(n-2)$ if $n \geq 3)$ and $f(u)=u \log |u|$ are treated. The $L^{2}$ and $H^{1}$ norm estimates $C_{1}(k+1)^{n / 2} \leq\|u\|_{L^{2}} \leq\|u\|_{H^{1}} \leq C_{2}(k+1)^{n / 2}$ are established for any radially symmetric solution $u$ which has exactly $k \geq 0$ zeros in the interval $0 \leq|x|<\infty$. Here $C_{1}, C_{2}>0$ are independent of $u$ and $k$.
\end{abstract}

\section{INTRODUCTION}

In this paper we give the $L^{2}\left(\mathbf{R}^{n}\right)$ and $H^{1}\left(\mathbf{R}^{n}\right)$ norm estimates for radially symmetric solutions of the semilinear elliptic problem

$$
\begin{aligned}
\Delta u+f(u) & =0, \quad x \in \mathbf{R}^{n}, \\
\lim _{|x| \rightarrow \infty} u(x) & =0,
\end{aligned}
$$

where $n \geq 2$ and $f(u)$ is a continuous function. We are interested in the power nonlinearity $f(u)=|u|^{p-1} u-u$ and the logarithmic nonlinearity $f(u)=$ $u \log |u|$. The solution of the problem (1.1)-(1.2) is considered as a bound state of the nonlinear Schrödinger equation

$$
-i \frac{\partial v}{\partial t}=\Delta v+g(v)
$$

or a standing wave solution of the nonlinear Klein-Gordon equation

$$
\frac{\partial^{2} v}{\partial t^{2}}=\Delta v-m^{2} v+g(v)
$$

Here $g(v)$ is a continuous function from $\mathbf{C}$ to $\mathbf{C}$ satisfying

$$
g\left(r e^{i \theta}\right)=e^{i \theta} g(r) \quad(r, \theta \in \mathbf{R}), \quad g(\mathbf{R}) \subset \mathbf{R} .
$$

In equation (1.3) or (1.4), the solution $v$ of the form $v(x, t)=e^{i \lambda t} w(x)$ is called a bound state or a standing wave solution if $w \not \equiv 0$ is a square integrable

Received by the editors April 16, 1993.

1991 Mathematics Subject Classification. Primary 35J60; Secondary 34B15.

Key words and phrases. Semilinear elliptic equation, radially symmetric solution, norm estimate. 
real-valued function. Then equation (1.3) is reduced to the semilinear elliptic equation

$$
\triangle w+g(w)-\lambda w=0,
$$

which becomes equation (1.1) by setting $f(w)=g(w)-\lambda w$. Equation (1.4) is also rewritten as equation (1.6) in which $\lambda$ is replaced by $\lambda^{2}-m^{2}$. Condition (1.2) is caused by $w \in L^{2}\left(\mathbf{R}^{n}\right)$. In equation (1.6) with a special type of the nonlinear term $g(w)=|w|^{p-1} w$ or $w \log |w|$, we employ the the change of variable $w(x)=\lambda^{1 /(p-1)} u(\sqrt{\lambda} x)$ or $w(x)=e^{\lambda} u(x)$, respectively. Then equation (1.6) is transformed into the equation

$$
\triangle u+|u|^{p-1} u-u=0
$$

or

$$
\triangle u+u \log |u|=0 .
$$

It has been proved by many authors $[1,2,4,7,8,10]$ that under suitable assumptions on $f(u)$, for example, $f(u)=|u|^{p-1} u-u$ with $1<p<$ $(n+2) /(n-2)$ or $f(u)=u \log |u|$, equation (1.1)-(1.2) has infinitely many radially symmetric solutions. More precisely, (see Grillakis [2] and Kajikiya [7]) equation (1.1)-(1.2) possesses a sequence $\left\{u_{k}\right\}_{k=0}^{\infty} \subset C^{2}\left(\mathbf{R}^{n}\right) \cap H^{1}\left(\mathbf{R}^{n}\right)$ of radially symmetric solutions such that $u_{k}$ has exactly $k$ zeros in the interval $0 \leq|x|<\infty$ and

$$
\lim _{k \rightarrow \infty}\left\|u_{k}\right\|_{H^{1}}=\lim _{k \rightarrow \infty} L\left(u_{k}\right)=+\infty .
$$

Here $H^{1}\left(\mathbf{R}^{n}\right)$ and $\|\cdot\|_{H^{1}}$ denote the usual $L^{2}$ Sobolev space of the first order and the corresponding norm, respectively. The functional $L(u)$ stands for the Lagrangian of (1.1), i.e.,

$$
L(u)=\int_{\mathbf{R}^{n}}\left(\frac{1}{2}|\nabla u|^{2}-F(u)\right) d x, \quad \text { where } \quad F(u)=\int_{0}^{u} f(s) d s .
$$

The above result raises a question: What is the relation between the Sobolev norm or the Lagrangian of radially symmetric solutions and the number of their zeros? The purpose of this paper is to solve the question. Our answer is as follows. Any radially symmetric solution $u$ of (1.7) or (1.8) with (1.2) is estimated as

$$
\begin{gathered}
C_{1}(k+1)^{n / 2} \leq\|u\|_{L^{2}} \leq\|u\|_{H^{1}} \leq C_{2}(k+1)^{n / 2}, \\
C_{3}(k+1)^{n} \leq L(u) \leq C_{4}(k+1)^{n},
\end{gathered}
$$

provided that $u$ has exactly $k(\geq 0)$ zeros in the interval $0 \leq|x|<\infty$. Here $\|u\|_{L^{2}}$ denotes the $L^{2}$-norm of $u$. Each $C_{i}$ is a positive constant independent of $u$ and $k$. It will be proved later that any radially symmetric solution of (1.7) or (1.8) with (1.2) has at most a finite number of zeros.

To obtain the estimates $(1.10)$ and (1.11), this paper is organized into six sections. In Section 2, we impose several assumptions on the nonlinear term $f(u)$ and state our theorem strictly. In Section 3, we prepare some technical lemmas which will play an important role in the subsequent sections. In Section 4 , we investigate the various properties of radially symmetric solutions. In Section 5, we give the lower estimate for the Sobolev norms of solutions via 
the number of zeros. In Section 6, we prove the upper estimate for the Sobolev norms of solutions.

\section{MAIN RESULT}

In this section, we state the main result. In order to discuss radially symmetric solutions $u=u(r), r=|x|$, we convert (1.1)-(1.2) to the singular boundary value problem for the ordinary differential equation

$$
\begin{aligned}
& u^{\prime \prime}+\frac{n-1}{r} u^{\prime}+f(u)=0, \quad r>0, \\
& u^{\prime}(0)=0, \quad \lim _{r \rightarrow \infty} u(r)=0 .
\end{aligned}
$$

In the following, we impose several assumptions on the function $f(s)$.

(f1) Suppose that $f(s)$ is continuous and that there exists a unique local solution to any initial value problem for equation (2.1).

It is well-known that assumption (f1) is fulfilled if $f(s)$ is locally Lipschitz continuous. The function $f(u)=u \log |u|$ in (1.8) is not locally Lipschitz continuous, however, condition (f1) is satisfied. This has been proved in [7, Appendix]. Next, we impose conditions on the sign of $f(s)$ and on the behavior of $f(s)$ as $s \rightarrow 0$.

(f2) There exist positive constants $a$ and $b$ such that

$$
f(s)>0, \quad s \in(-b, 0) \cup(a, \infty), \quad f(s)<0, \quad s \in(-\infty,-b) \cup(0, a) .
$$

(f3) There exists a constant $q \geq 1$ such that

$$
-\infty \leq \limsup _{s \rightarrow 0} \frac{f(s)}{|s|^{q-1} s}<0 \text { and } \limsup _{s \rightarrow 0}\left|\frac{s f(s)}{F(s)}\right|<\infty,
$$

where $F(s)=\int_{0}^{s} f(r) d r$.

The following assumptions imply that $f(s)$ has a superlinear and subcritical growth order in the neighborhood of $s= \pm \infty$.

$$
\begin{gathered}
\lim _{s \rightarrow \pm \infty} \frac{f(s)}{s}=\infty . \\
0<\liminf _{s \rightarrow \pm \infty} \frac{s f(s)}{F(s)} \leq \limsup _{s \rightarrow \pm \infty} \frac{s f(s)}{F(s)}<\infty \quad \text { if } n=2, \\
0<\liminf _{s \rightarrow \pm \infty} \frac{s f(s)}{F(s)} \leq \limsup _{s \rightarrow \pm \infty} \frac{s f(s)}{F(s)}<\frac{2 n}{n-2} \quad \text { if } n \geq 3 .
\end{gathered}
$$

It is easy to check that all of the conditions (f1)-(f5) are satisfied by the functions, for example, $f(s)=|s|^{p-1} s-s, f(s)=|s|^{p-1} s-|s|^{q-1} s$, where $1<q<p<\infty$ if $n=2$ and $1<q<p<(n+2) /(n-2)$ if $n \geq 3$, and $f(s)=s \log |s|$. Before stating the main theorem, we present our earlier result which guarantees the existence of solutions.

Theorem 0 ([7, Theorem 4]). Suppose that assumptions (f1), (f2), (f4) and (f5) hold. Then there exist sequences $\left\{u_{k}\right\}_{k=0}^{\infty}$ and $\left\{v_{k}\right\}_{k=0}^{\infty}$ of solutions to (2.1)(2.2) such that $u_{k}$ and $v_{k}$ have exactly $k(\geq 0)$ zeros in $(0, \infty)$ and $u_{k}(0)>$ $0>v_{k}(0)$. 
For any solution $u$ of $(2.1)-(2.2)$, it will be proved later on that $\nabla u \in L^{2}\left(\mathbf{R}^{n}\right)$ and $F(u) \in L^{1}\left(\mathbf{R}^{n}\right)$ and moreover that $u$ has at most a finite number of zeros in $(0, \infty)$. Therefore the main result below makes sense.

Theorem 1. Under assumptions (f1)-(f5), there exist positive constants $C_{i} \quad(1 \leq$ $i \leq 4)$ such that

$$
C_{1}(k+1)^{n / 2} \leq\|\nabla u\|_{L^{2}} \leq C_{2}(k+1)^{n / 2}, \quad C_{3}(k+1)^{n} \leq L(u) \leq C_{4}(k+1)^{n}
$$

for any solution $u$ of (2.1)-(2.2) which has exactly $k(\geq 0)$ zeros. Here $L(u)$ is defined by (1.9). Moreover, if $f(u)=|u|^{p-1} u-u$, where $1<p<\infty$ when $n=2$ and $1<p<(n+2) /(n-2)$ when $n \geq 3$, or if $f(u)=u \log |u|$, then we obtain the estimate

$$
C_{5}(k+1)^{n / 2} \leq\|u\|_{L^{2}} \leq\|u\|_{H^{1}} \leq C_{6}(k+1)^{n / 2} .
$$

Here $C_{5}$ and $C_{6}$ are also positive constants independent of $u$ and $k$.

From the above result we see that the $H^{1}$-norm of solution grows like $k^{n / 2}$ as $k \rightarrow \infty$. The growth rate $n / 2$ depends only on the dimension $n$, does not on the growth order of $f(u)$ as $u \rightarrow \pm \infty$.

On the other hand, in $[5,6]$ we have studied equation $(2.1)$ in the unit ball:

$$
\begin{aligned}
u^{\prime \prime}+\frac{n-1}{r} u^{\prime}+f(u) & =0, \quad 0<r<1, \\
u^{\prime}(0)=u(1) & =0,
\end{aligned}
$$

where $f(u)$ behaves like $|u|^{p-1} u$ as $u \rightarrow \pm \infty$ and $1<p<\infty$ for $n=2$ and $1<p<(n+2) /(n-2)$ for $n \geq 3$. Then we have obtained the estimate

$$
C_{1}(k+1)^{(p+1) /(p-1)} \leq\|u\|_{H^{1}} \leq C_{2}(k+1)^{(p+1) /(p-1)}
$$

for any solution $u$ of $(2.3)-(2.4)$ with $k$ zeros in $(0,1)$. Here $\|u\|_{H^{1}}$ denotes the $H^{1}$-norm of $u$ on the unit ball and $C_{1}$ and $C_{2}$ are independent of $u$ and $k$. In this case, the growth rate $(p+1) /(p-1)$ depends only on the growth order of $f(u)$, does not on the dimension $n$.

\section{Preliminary lemmas}

From here to the end of the paper, we always suppose that all of assumptions (f1) through (f5) hold. In this section, we prepare some technical but crucial lemmas. Let $F(s)$ be defined in assumption (f3), that is,

$$
F(s)=\int_{0}^{s} f(r) d r
$$

Definition 3.1. By assumptions (f2) and (f4) the function $F(s)$ has exactly two zeros except for $s=0$. We denote these zeros by $-B$ and $A$. That is,

$$
-B<-b<0<a<A \quad \text { and } \quad F(A)=F(-B)=0 .
$$

From the above definition it follows that

$$
\begin{array}{cl} 
& F(A)=F(-B)=F(0)=0, \\
F(s)<0 & \text { for } \quad s \in(-B, A) \backslash\{0\}, \\
F(s)>0 & \text { for } \quad s \in(-\infty,-B) \cup(A, \infty) .
\end{array}
$$


In what follows, we present three lemmas which will be used in Sections 5 and 6.

Lemma 3.2. For any $A_{1}$ in $(A, \infty)$ and any $-B_{1}$ in $(-\infty,-B)$ there exists $a$ constant $\theta_{0} \in(0,1)$ such that

$$
F(s) \leq 2 F(\theta s) \leq 2 F(s) \text { when } \theta s \in\left(-\infty,-B_{1}\right] \cup\left[A_{1}, \infty\right) \text { and } \theta \in\left(\theta_{0}, 1\right) \text {. }
$$

Proof. We show that there exists a positive constant $C$ depending only on $A_{1}$ and $B_{1}$ such that

$$
(1+C \log \theta) F(s) \leq F(\theta s)
$$

provided that $\theta \in(0,1)$ and $\theta s \in\left(-\infty,-B_{1}\right] \cup\left[A_{1}, \infty\right)$.

By assumption (f5) together with (3.2) there exists a constant $C>0$ such that $s f(s) \leq C F(s)$ for $s \in\left(-\infty,-B_{1}\right] \cup\left[A_{1}, \infty\right)$. Let $\theta \in(0,1)$ and $\theta s \geq A_{1}>0$. Then we have

$$
F(s)=F(\theta s)+\int_{\theta s}^{s} f(r) d r \leq F(\theta s)+C \int_{\theta s}^{s} \frac{F(r)}{r} d r .
$$

Since $F(r)$ is strictly increasing in $(a, \infty)$ by (f2), the last integral is estimated as

Therefore we obtain

$$
\int_{\theta s}^{s} \frac{F(r)}{r} d r \leq-F(s) \log \theta .
$$

$$
F(s) \leq F(\theta s)-C(\log \theta) F(s) .
$$

The same argument as above is also valid for $\theta s \in\left(-\infty,-B_{1}\right]$. Thus we have (3.3). In the inequality (3.3) we choose $\theta_{0}$ to be sufficiently closed to 1 such that $-1 / 2<C \log \theta_{0}<0$. Then we obtain the first inequality of the lemma. The second one follows from the monotonicity of $F(s)$. Hence the proof is complete.

Lemma 3.3. Let $A_{1} \in(A, \infty)$ and $-B_{1} \in(-\infty,-B)$. Then there exist positive constants $C_{1}, C_{2}$ and $p$ such that

$$
\begin{gathered}
1<p<\infty \text { when } n=2, \quad 1<p<\frac{n+2}{n-2} \text { when } n \geq 3, \\
s f(s) \leq C_{1} F(s) \leq C_{2}|s|^{p+1} \quad \text { for } s \in\left(-\infty,-B_{1}\right] \cup\left[A_{1}, \infty\right) .
\end{gathered}
$$

Proof. We choose a suitable constant $p>1$ by (f5) such that

$$
\begin{array}{ll}
\limsup _{s \rightarrow \pm \infty} \frac{s f(s)}{F(s)}<p+1<\infty & \text { if } n=2, \\
\limsup _{s \rightarrow \pm \infty} \frac{s f(s)}{F(s)}<p+1<\frac{2 n}{n-2} & \text { if } n \geq 3 .
\end{array}
$$

In other words, there exists an $R>0$ such that

$$
\frac{s F^{\prime}(s)}{F(s)}=\frac{s f(s)}{F(s)}<p+1 \text { for }|s| \geq R .
$$

Solving the differential inequality, we obtain (3.4) for $|s| \geq R$. For $s$ in $\left[-R,-B_{1}\right] \cup\left[A_{1}, R\right]$, we use $F(s)>0$ to get (3.4). This completes the proof.

In Section 5, we use a nonlinear variant of Prüfer transformation to estimate the Sobolev norms of solutions. To do so, we need the next lemma. 
Lemma 3.4. Let $-b_{1}$ and $a_{1}$ be fixed in $(-B,-b)$ and in $(a, A)$, respectively. Then there exist two continuous functions $g(s)$ and $h(s)$ satisfying the following conditions.

(i) $f(s)=g(s)-h(s)$ for $s \in \mathbf{R}, \quad f(s)=g(s)$ for $s \in\left(-\infty,-b_{1}\right] \cup$ $\left[a_{1}, \infty\right)$.

(ii) It holds that $s g(s), G(s), H(s)>0$ for any $s \neq 0$, where

$$
G(s)=\int_{0}^{s} g(r) d r \text { and } H(s)=\int_{0}^{s} h(r) d r .
$$

(iii) The function $f(s) / g(s)$ is bounded above.

(iv) $\frac{1}{2} H(s) \geq h(s) \frac{G(s)}{g(s)}$ for $s \in \mathbf{R}$.

Proof. By assumption (f3) there exist positive constants $C, \delta$ and $r$ such that

$$
C|F(s)| \geq|s f(s)| \text { and } F(s) \leq-\delta|s|^{q+1} \text { for } s \in(-r, r) \text {. }
$$

Since $|F(s)|=-F(s)>0$ for $s \in\left[-b_{1}, a_{1}\right] \backslash\{0\}$ by (3.2), the above inequality gives a constant $C_{0}$ such that

$$
C_{0}|F(s)| \geq|s f(s)| \text { for } s \in\left[-b_{1}, a_{1}\right]
$$

and

$$
C_{0}|F(s)| \geq|s|^{q+1} \quad \text { for } \quad s \in\left[-b_{1}, a_{1}\right] .
$$

We now choose $\mu$ so large that

$$
\mu>2 q-1 \text { and } \frac{\mu+1}{2 C_{0}}>\max \left\{f\left(a_{1}\right) a_{1}^{-q},\left|f\left(-b_{1}\right)\right| b_{1}^{-q}, 2\right\} .
$$

We moreover define $\bar{a}, \bar{b}>0$ by

$$
\bar{a}=f\left(a_{1}\right) a_{1}^{-\mu} \quad \text { and } \quad \bar{b}=-f\left(-b_{1}\right) b_{1}^{-\mu} .
$$

Then we define the function $g(s)$ by

$$
g(s)= \begin{cases}f(s), & s \in\left(-\infty,-b_{1}\right] \cup\left[a_{1}, \infty\right), \\ \bar{b}|s|^{\mu-1} s, & s \in\left[-b_{1}, 0\right], \\ \bar{a}|s|^{\mu-1} s, & s \in\left[0, a_{1}\right] .\end{cases}
$$

We set $h(s)=g(s)-f(s)$. Then it is clear that $g$ and $h$ are continuous and satisfy condition (i). From the definition of $g(s)$ it follows that $s g(s), G(s)>0$ for $s \neq 0$. Noting that $H(s)=G(s)-F(s)$ and $F(s)<0$ for $s \in\left(0, a_{1}\right]$, we see that $H(s)>0$ for $s \in\left(0, a_{1}\right]$. Since $H^{\prime}(s)=h(s)=0 \quad\left(s \geq a_{1}\right)$, it holds that $H(s)=H\left(a_{1}\right)>0$ for $s \geq a_{1}$. Thus we have that $H(s)>0$ for $s>0$. The same argument implies that $H(s)>0$ for $s<0$ as well.

We prove assertion (iii). From the definition of $g(s)$ it follows that $f(s) / g(s)$ $<0$ for $s \in(0, a)$ and $f(s) / g(s)=1$ for $s \in\left[a_{1}, \infty\right)$. Since $g(s)>0$ on $\left[a, a_{1}\right]$, the function $f(s) / g(s)$ is bounded above on $(0, \infty)$. The boundedness above on $(-\infty, 0)$ follows from the same argument as above.

We show assertion (iv). Since $H(s)>0, h(s)=0$ for $s \in\left(-\infty,-b_{1}\right] \cup$ $\left[a_{1}, \infty\right)$, the inequality (iv) holds for $s$ in these intervals. Let $s \in\left(0, a_{1}\right)$. Then we use (3.9) to rewrite inequality (iv) as

$$
\frac{1}{2}\left(\bar{a} s^{\mu+1}-(\mu+1) F(s)\right) \geq \bar{a} s^{\mu+1}-s f(s) .
$$


We note that $|F(s)|=-F(s)>0$. Therefore

$$
(\mu+1)|F(s)| \geq \bar{a} s^{\mu+1}-2 s f(s) \text { for } s \in\left(0, a_{1}\right) .
$$

To show this, it is sufficient to prove the inequalities

$$
\frac{\mu+1}{2}|F(s)| \geq \bar{a} s^{\mu+1}
$$

and

$$
\frac{\mu+1}{2}|F(s)| \geq 2|s f(s)|
$$

for $s \in\left(0, a_{1}\right)$. The above inequality (3.12) follows readily from (3.5) and (3.7). We show (3.11). It follows from (3.7) and (3.8) that

$$
\bar{a} s^{\mu+1} \leq \bar{a} a_{1}^{\mu-q} s^{q+1}=f\left(a_{1}\right) a_{1}^{-q} s^{q+1} \leq \frac{\mu+1}{2 C_{0}} s^{q+1} \text { for } s \in\left(0, a_{1}\right) .
$$

This together with (3.6) yields (3.11). Therefore we obtain (3.10) and inequality (iv) for $s \geq 0$. The same method as above remains valid for $s \leq 0$. The proof is thereby complete.

\section{Property of Solutions}

In this section, we investigate the property of solutions in detail. We first consider the initial value problem for (2.1) at $r=0$,

$$
\begin{aligned}
u^{\prime \prime}+\frac{n-1}{r} u^{\prime}+f(u) & =0, \quad r>0, \\
u^{\prime}(0)=0, \quad u(0) & =\lambda .
\end{aligned}
$$

Definition 4.1. We denote the solution of $(4.1)-(4.2)$ by $u(r, \lambda)$.

We prove the global existence of the solution $u(r, \lambda)$ and the continuous dependence on the initial data $\lambda$.

Lemma 4.2. (i) For any $\lambda \in \mathbf{R}$, the solution $u(r, \lambda)$ can be extended to $r=\infty$. Moreover, we have for any $\Lambda>0$,

$$
\sup \left\{|u(r, \lambda)|+\left|u^{\prime}(r, \lambda)\right|+\left|u^{\prime \prime}(r, \lambda)\right|: 0 \leq r<\infty, \quad|\lambda| \leq \Lambda\right\}<\infty .
$$

(ii) The solution depends continuously on $\lambda$. More precisely, for any $\lambda_{0} \in \mathbf{R}$ and $R>0$, the solution $u(\cdot, \lambda)$ converges to $u\left(\cdot, \lambda_{0}\right)$ in $C^{2}[0, R]$ as $\lambda \rightarrow \lambda_{0}$. Proof. This lemma has been proved in our earlier paper [7, Lemma 3.1]; however, for the sake of completeness we give the proof in the following. Let $\Lambda>0$ be given and $\lambda$ satisfy $|\lambda| \leq \Lambda$. The local solution $u(r)=u(r, \lambda)$ of $(4.1)-(4.2)$ is uniquely defined because of assumption (f1). We define the energy function $E(r)$ by

$$
\begin{aligned}
& E(r)=E(r, \lambda) \equiv \frac{1}{2} u^{\prime}(r, \lambda)^{2}+F(u(r, \lambda)), \\
& F(s)=\int_{0}^{s} f(\tau) d \tau .
\end{aligned}
$$

From an easy computation it follows that

$$
E^{\prime}(r)=-\frac{n-1}{r} u^{\prime}(r)^{2} \leq 0,
$$


which implies that $E(r)$ is decreasing. Moreover, if $u(r)$ is not a stationary solution, then the zeros of $u^{\prime}(r)$ are isolated and therefore $E(r)$ is strictly decreasing. Hence we have

$$
\frac{1}{2} u^{\prime}(r)^{2}+F(u(r))=E(r) \leq E(0) \leq \max _{|\lambda| \leq \Lambda} F(\lambda) .
$$

Noting that $\lim _{s \rightarrow \pm \infty} F(s)=\infty$ by (f4), we have a constant $C_{\Lambda}>0$ such that

$$
|u(r, \lambda)|, \quad\left|u^{\prime}(r, \lambda)\right| \leq C_{\Lambda} \quad \text { for } r \geq 0 \text { and }|\lambda| \leq \Lambda,
$$

which implies that $u$ can be extended to $r=\infty$.

We show $u^{\prime \prime}$ to be bounded. Equation (4.1) is rewritten as

$$
\left(r^{n-1} u^{\prime}\right)^{\prime}=-r^{n-1} f(u)
$$

Integrating this relation over $[0, r]$, we get

$$
r^{n-1} u^{\prime}(r)=-\int_{0}^{r} f(u(s)) s^{n-1} d s,
$$

which together with (4.5) implies

$$
\left|u^{\prime}(r)\right| \leq \frac{C_{1}}{n} r, \quad \text { where } \quad C_{1}=\max _{|s| \leq C_{\Lambda}}|f(s)| .
$$

Using this inequality in (4.1), we obtain

$$
\left|u^{\prime \prime}(r)\right| \leq \frac{n-1}{r}\left|u^{\prime}\right|+|f(u)| \leq \frac{n-1}{n} C_{1}+C_{1}
$$

for $r \geq 0$ and $|\lambda| \leq \Lambda$. This inequality and (4.5) prove assertion (i).

We prove assertion (ii). Let $R>0$ and $\lambda_{0} \in \mathbf{R}$ be given. Let $\left\{\lambda_{i}\right\}$ be a sequence converging to $\lambda_{0}$. We write $u_{i}(r)=u\left(r, \lambda_{i}\right)$ for $i \geq 0$. Instead of showing the convergence of $\left\{u_{i}\right\}$ to $u_{0}$ in $C^{2}[0, R]$, we will prove the equivalent statement: For any subsequence $\left\{v_{j}\right\}$ of $\left\{u_{i}\right\}$, there exists a subsequence $\left\{w_{k}\right\}$ of $\left\{v_{j}\right\}$ such that $w_{k}$ converges to $u_{0}$ in $C^{2}[0, R]$.

Let $\left\{v_{j}\right\}$ be any subsequence of $\left\{u_{i}\right\}$. Applying Ascoli-Arzela's theorem together with assertion (i), we choose a subsequence $\left\{w_{k}\right\}$ of $\left\{v_{j}\right\}$ such that $w_{k}$ converges to a certain limit $w_{0}$ in $C^{1}[0, R]$. We now recall that $w_{k}$ satisfies (4.7), that is,

$$
r^{n-1} w_{k}^{\prime}(r)=-\int_{0}^{r} f\left(w_{k}(s)\right) s^{n-1} d s .
$$

Letting $k \rightarrow \infty$, we have

$$
r^{n-1} w_{0}^{\prime}(r)=-\int_{0}^{r} f\left(w_{0}(s)\right) s^{n-1} d s
$$

which implies that $w_{0} \in C^{2}(0, R]$ and $w_{0}$ satisfies (4.1). Since $w_{0}^{\prime}(0)=$ $\lim _{k \rightarrow \infty} w_{k}^{\prime}(0)=0$ and $w_{0}(0)=\lim _{k \rightarrow \infty} w_{k}(0)=\lambda_{0}$, it follows from (f1) that $w_{0}(r) \equiv u_{0}(r)$. We prove that $w_{k}^{\prime \prime}$ converges uniformly to $u_{0}^{\prime \prime}=w_{0}^{\prime \prime}$. The identities (4.9) and (4.10) yield

$$
\frac{1}{r}\left|w_{k}^{\prime}(r)-w_{0}^{\prime}(r)\right| \leq n^{-1} \max _{0 \leq s \leq R}\left|f\left(w_{k}(s)\right)-f\left(w_{0}(s)\right)\right| .
$$


Therefore $(1 / r) w_{k}^{\prime}(r)$ converges uniformly on $[0, R]$, so does the right-hand side of

$$
w_{k}^{\prime \prime}=-\frac{n-1}{r} w_{k}^{\prime}-f\left(w_{k}(r)\right) \text {. }
$$

Thus $w_{k}$ converges to $w_{0}=u_{0}$ in $C^{2}[0, R]$ and this completes the proof.

The next lemma gives us information about zeros, zero derivatives and local extrema of solutions.

Lemma 4.3. Let $u$ satisfy $(4.1)$ on $[0, \infty)$ and be not a stationary solution. Then we have:

(i) If $u^{\prime}\left(r_{0}\right)=0$ for some $r_{0}$, then $u^{\prime \prime}\left(r_{0}\right) \neq 0$ and $u(r)$ has a strict local maximum or minimum at $r_{0}$.

(ii) If $u(r)$ has a local maximum (or minimum) at $r_{0}$, then $u(r)<u\left(r_{0}\right)$ (or $\left.u(r)>u\left(r_{0}\right)\right)$ for all $r \in\left(r_{0}, \infty\right)$.

(iii) If $u\left(r_{1}\right)=u\left(r_{2}\right)=0$ and $u(r)>0$ (or $<0$ ) for $r \in\left(r_{1}, r_{2}\right)$ with some $r_{1}, r_{2}$, then there exists a unique $\xi \in\left(r_{1}, r_{2}\right)$ such that $u^{\prime}(\xi)=0$. Furthermore, it holds that $u(\xi)>A$ (or $u(\xi)<-B$ ) and that $u^{\prime}(r)>0$ (or $\left.<0\right)$ for $r \in\left[r_{1}, \xi\right)$ and $u^{\prime}(r)<0$ (or $\left.>0\right)$ for $r \in\left(\xi, r_{2}\right]$.

Proof. If $u^{\prime}\left(r_{0}\right)=u^{\prime \prime}\left(r_{0}\right)=0$ for some $r_{0} \geq 0$, then $f\left(u\left(r_{0}\right)\right)=0$ by (4.1), hence $u(r) \equiv u\left(r_{0}\right)$ is a stationary solution. This contradicts the assumption of the lemma. Therefore we have assertion (i).

To prove (ii), it is sufficient to show that

$$
u(r) \neq u\left(r_{0}\right) \quad \text { for } \quad r \in\left(r_{0}, \infty\right) \quad \text { if } \quad u^{\prime}\left(r_{0}\right)=0 .
$$

Since $E(r)$ is strictly decreasing and $u^{\prime}\left(r_{0}\right)=0$, we have $F\left(u\left(r_{0}\right)\right)=E\left(r_{0}\right)>$ $E(r) \geq F(u(r))$ for $r>r_{0}$. Hence $u\left(r_{0}\right) \neq u(r)$.

We prove (iii). Let $u\left(r_{1}\right)=u\left(r_{2}\right)=0$ and $u(r)>0$ for $r \in\left(r_{1}, r_{2}\right)$. By Rolle's theorem there exists a point $\xi \in\left(r_{1}, r_{2}\right)$ such that $u^{\prime}(\xi)=0$. On the other hand, since $u(r)$ has only simple zeros and $E(r)$ is strictly decreasing, we have

$$
E(r)>E\left(r_{2}\right)=\frac{1}{2} u^{\prime}\left(r_{2}\right)^{2}>0 \quad \text { for } \quad r \in\left(r_{1}, r_{2}\right) .
$$

Then it follows that $0<E(\xi)=F(u(\xi))$. Therefore our assumption $u(r)>$ 0 implies that $u(\xi)>A$. We substitute $r=\xi$ into (4.1) to get $u^{\prime \prime}(\xi)=$ $-f(u(\xi))<0$ since $u(\xi)>A$. Hence we obtain the fact that $u^{\prime \prime}(\xi)<0$ if $u^{\prime}(\xi)=0$ at some $\xi \in\left(r_{1}, r_{2}\right)$. This fact proves the uniqueness of zeros of $u^{\prime}$ and moreover that $u^{\prime}(r)>0$ for $r \in\left[r_{1}, \xi\right)$ and $u^{\prime}(r)<0$ for $r \in\left(\xi, r_{2}\right]$. The proof is thereby complete.

In the following lemma, we investigate the asymptotic behavior of solutions to (2.1)-(2.2) as $r \rightarrow \infty$ and proves the decay of the energy. Moreover we show that $\nabla u \in L^{2}\left(\mathbf{R}^{n}\right)$ and $F(u) \in L^{1}\left(\mathbf{R}^{n}\right)$, therefore the Lagrangian $L(u)$ is well-defined and Theorem 1 makes sense.

Lemma 4.4. Let $u \not \equiv 0$ be a solution of $(4.1)$ on $[0, \infty)$ which converges to 0 as $r \rightarrow \infty$. Denote by $E(r)$ the energy associated with $u$. Then the following assertions hold :

(i) $u$ has at most a finite number of zeros in $[0, \infty)$.

(ii) $\lim _{r \rightarrow \infty} u^{\prime}(r)=\lim _{r \rightarrow \infty} u^{\prime \prime}(r)=\lim _{r \rightarrow \infty} E(r)=0$ and $E(r)>0$ for $r \geq 0$.

(iii) $\nabla u \in L^{2}\left(\mathbf{R}^{n}\right)$ and $u f(u), F(u) \in L^{1}\left(\mathbf{R}^{n}\right)$. 
(iv) $\sup _{r \geq 0}\left|u^{\prime}(r)\right| r^{n-1}<\infty$ and $\lim _{r \rightarrow \infty} E(r) r^{n}=0$

Proof. Assertion (i) readily follows from Lemma 4.3 (iii) since $u(r)$ tends to 0 as $r \rightarrow \infty$. We prove assertion (ii). Since $F(s)$ is bounded below, so is $E(r)$. Recall that $E(r)$ is strictly decreasing, hence it has a certain limit $E_{\infty}$ as $r \rightarrow \infty$. Then we have

$$
\frac{1}{2} u^{\prime}(r)^{2}=E(r)-F(u(r)) \longrightarrow E_{\infty} \quad \text { as } \quad r \rightarrow \infty,
$$

which implies that $E_{\infty} \geq 0$ and $\lim _{r \rightarrow \infty}\left|u^{\prime}(r)\right|=\sqrt{2 E_{\infty}}$. If $E_{\infty}>0$, then $\lim _{r \rightarrow \infty} u^{\prime}(r)=\sqrt{2 E_{\infty}}$ or $-\sqrt{2 E_{\infty}}$. But this contradicts the boundedness of $u(r)$. Thus it follows that $E_{\infty}=0$ and $\lim _{r \rightarrow \infty} u^{\prime}(r)=0$. Moreover it holds that $E(r)>0$ for $r \geq 0$ since $E(r)$ is strictly decreasing. Letting $r \rightarrow \infty$ in (4.1), we see that $\lim _{r \rightarrow \infty} u^{\prime \prime}(r)=0$. that

We prove assertion (iii). By assertion (i) there exists a constant $R>0$ such

$$
0<u(r)<a \quad(r \geq R)
$$

or

$$
-b<u(r)<0 \quad(r \geq R)
$$

We deal with case (A) only. However case (B) can be treated in the same way. Assertion (ii) means

$$
E(r)=\frac{1}{2} u^{\prime}(r)^{2}+F(u(r))>0 .
$$

Since $F(u(r))<0$ in case $(\mathrm{A})$, it holds that $u^{\prime}(r)^{2}>0$, and so $u^{\prime}(r)<0$ for $r \geq R$.

On the other hand, we rewrite (4.1) as

$$
\left(r^{n-1} u^{\prime}\right)^{\prime}=-r^{n-1} f(u) \text {. }
$$

The right-hand side is positive because of $(\mathrm{A})$. Therefore $r^{n-1} u^{\prime}(r)(<0)$ is increasing and $\left|u^{\prime}(r)\right| r^{n-1}$ is decreasing in $[R, \infty)$. Hence we have

$$
\left|u^{\prime}(r)\right| r^{n-1} \leq C_{0} \equiv\left|u^{\prime}(R)\right| R^{n-1} \quad \text { for } \quad r \geq R .
$$

This inequality gives

$$
\int_{R}^{s} u^{\prime}(r)^{2} r^{n-1} d r \leq C_{0} \int_{R}^{s}\left|u^{\prime}(r)\right| d r=-C_{0} \int_{R}^{s} u^{\prime}(r) d r \leq C_{0} u(R) .
$$

Letting $s \rightarrow \infty$, we deduce that

$$
\int_{R}^{\infty} u^{\prime}(r)^{2} r^{n-1} d r \leq C_{0} u(R)<\infty
$$

Thus we obtain $\nabla u \in L^{2}\left(\mathbf{R}^{r}\right)$.

We show that $u f(u) \in L^{1}\left(\mathbf{R}^{n}\right)$. Multiplying (4.1) by $u(r) r^{n-1}$ and integrating it over $[R, r]$, we obtain

$$
\begin{aligned}
-\int_{R}^{r} u f(u) s^{n-1} d s & =-\int_{R}^{r}\left|u^{\prime}\right|^{2} s^{n-1} d s+u^{\prime}(r) u(r) r^{n-1}-u^{\prime}(R) u(R) R^{n-1} \\
& \leq-u^{\prime}(R) u(R) R^{n-1}
\end{aligned}
$$


Here we have used that $u^{\prime}(r)<0$ and $u(r)>0$ for $r \geq R$ in case (A). Since $|u f(u)|=-u f(u)$ for $0<u<a$, we have

$$
\int_{R}^{\infty}|u f(u)| s^{n-1} d s \leq\left|u^{\prime}(R) u(R)\right| R^{n-1} .
$$

We show that $F(u) \in L^{1}\left(\mathbf{K}^{n}\right)$. Note that $F(u)<0$ for $r \geq R$ in case (A). Then (4.11) implies

$$
|F(u(r))|=-F(u(r)) \leq \frac{1}{2} u^{\prime}(r)^{2} \quad \text { for } \quad r \geq R .
$$

Hence the assertion $F(u) \in L^{1}$ follows from $\nabla u \in L^{2}$.

Finally, we prove assertion (iv). From (4.12) it follows that $\sup _{r \geq 0}\left|u^{\prime}(r)\right| r^{n-1}$ $<\infty$. We show that $\lim _{r \rightarrow \infty} E(r) r^{n}=0$. Since $F(u(r))<0$ for $r \geq R$, we have that $0<E(r) \leq u^{\prime}(r)^{2} / 2$. Therefore it is sufficient to prove that $\lim _{r \rightarrow \infty} u^{\prime}(r)^{2} r^{n}=0$. If $n \geq 3$, it follows from (4.12) that

$$
u^{\prime}(r)^{2} r^{n} \leq C_{0}^{2} r^{-(n-2)} \longrightarrow 0 \quad \text { as } \quad r \rightarrow \infty .
$$

Let $n=2$. We want to show that $u^{\prime}(r)^{2} r^{n}=\left(u^{\prime}(r) r\right)^{2}$ tends to 0 as $r \rightarrow \infty$. Recall that $\left|u^{\prime}(r)\right| r$ is decreasing in $[R, \infty)$, which has been proved in the previous statement to (4.12) with $n=2$. Therefore we set $\delta=\lim _{r \rightarrow \infty}\left|u^{\prime}(r)\right| r$. If $\delta>0$, then $-u^{\prime}(r)=\left|u^{\prime}(r)\right| \geq \delta / r$ for $r \geq R$. Integrating both sides, we obtain

$$
-u(r)+u(R) \geq \delta \log (r / R) \longrightarrow \infty \quad \text { as } \quad r \rightarrow \infty,
$$

which contradicts that $\lim _{r \rightarrow \infty} u(r)=0$. Hence $\lim _{r \rightarrow \infty} u^{\prime}(r) r=0$. This completes the proof.

Definition 4.5. We denote by $S$ the set of all solutions $u \not \equiv 0$ of (2.1)-(2.2), that is,

$$
S=\left\{u \in C^{2}[0, \infty): u(r) \not \equiv 0 \text { satisfies }(2.1) \text { and }(2.2)\right\} .
$$

Moreover we define

$$
\begin{gathered}
S^{+}=\{u \in S: u(0)>0\}, \quad S^{-}=\{u \in S: u(0)<0\}, \\
S_{k}=\{u \in S: u \text { has exactly } k \text { zeros in }[0, \infty)\}, \\
S_{k}^{+}=S_{k} \cap S^{+} \text {and } S_{k}^{-}=S_{k} \cap S^{-} \text {for } k \geq 0 .
\end{gathered}
$$

We note that $S_{k}^{+}$and $S_{k}^{-}$are nonempty by Theorem 0 . Lemma 4.4 (i) implies that $S=\bigcup_{k=0}^{\infty} S_{k}$ and $S^{ \pm}=\bigcup_{k=0}^{\infty} S_{k}^{ \pm}$.

Combining Lemmas 4.3 and 4.4, we see the outline of the graph of solutions to $(2.1)-(2.2)$ in the following lemma.

Lemma 4.6. Let $u \in S_{k}^{+}$. If $k=0$, hence $u$ is a positive solution, then $u^{\prime}(r)<0$ for $r \in(0, \infty)$. If $k \geq 1$ and $\left\{r_{i}\right\}_{i=1}^{k}\left(0<r_{1}<r_{2}<\cdots<r_{k}\right)$ denote the zeros of $u$, then $u^{\prime}$ has exactly $k+1$ zeros $\left\{t_{i}\right\}_{i=0}^{k}$ in $[0, \infty)$ such that $0=t_{0}<r_{1}<t_{1}<\cdots<r_{k}<t_{k}$ and

$$
\begin{gathered}
u^{\prime}(r)<0 \text { for } r \in\left(t_{2 i}, t_{2 i+1}\right), \quad u^{\prime}(r)>0 \text { for } r \in\left(t_{2 i+1}, t_{2 i+2}\right), \\
(-1)^{k} u^{\prime}(r)<0 \text { for } r \in\left(t_{k}, \infty\right), \\
u\left(t_{2 i}\right)>A \text { and } u\left(t_{2 i+1}\right)<-B \text { for } i \geq 0 .
\end{gathered}
$$


The same result as above holds also for $u \in S_{k}^{-}$after exchanging $u^{\prime}(r)<0$ for $u^{\prime}(r)>0$ and replacing the last two inequalities by $u\left(t_{2 i}\right)<-B$ and $u\left(t_{2 i+1}\right)>$ $A$.

Proof. Let $u \in S_{0}^{+}$. Hence $u(r)$ is a positive solution of (4.1) which tends to 0 as $r \rightarrow \infty$. If $u^{\prime \prime}(0)>0$, then Lemma 4.3 (ii) implies that $u(r)>u(0)>0$ for all $r>0$. This contradicts that $\lim _{r \rightarrow \infty} u(r)=0$. Hence $u^{\prime \prime}(0)<0$, and so $u^{\prime}(r)<0$ for $r>0$ sufficiently small. If $u^{\prime}(T)=0$ at some first $T \in(0, \infty)$, then $u(r)$ has a local minimum at $r=T$. In fact, since $u^{\prime}(r)<0$ for $r<T$ and $u^{\prime}(T)=0$, it holds that $u^{\prime \prime}(T) \geq 0$, and therefore $u^{\prime \prime}(T)>0$ by Lemma 4.3 (i). Then it follows from Lemma 4.3 (ii) that $u(r)>u(T)>0$ for $r>T$, which contradicts that $\lim _{r \rightarrow \infty} u(r)=0$. Consequently, we see that $u^{\prime}(r)<0$ for all $r>0$. For $u \in S_{k}^{+}$also, the above discussion together with Lemma 4.3 proves Lemma 4.6.

We have already proved in Lemma 4.2 that for each $\lambda$, the energy $E(r, \lambda)$ is decreasing in $r$ and bounded below since $u(r, \lambda)$ and $u^{\prime}(r, \lambda)$ are also bounded. Therefore $E(r, \lambda)$ has a limit as $r \rightarrow \infty$.

Lemma 4.7. There exist constants $A_{*}$ and $B_{*}$ such that $A_{*}>A, B_{*}>B$ and

$$
\lim _{r \rightarrow \infty} E(r, \lambda)<0 \quad \text { for any } \lambda \in\left(-B_{*}, A_{*}\right) \backslash\{0\} .
$$

Proof. For $\lambda \in(-B, A) \backslash\{0\}$, we have

$$
E(r, \lambda) \leq E(0, \lambda)=F(\lambda)<0 \quad \text { for } \quad r \geq 0 .
$$

Hence, for such $\lambda$ the assertion holds. Let $\lambda=A$ and consider the energy $E(r, A)$. Since $u(r, A)$ is not a stationary solution, the corresponding energy is strictly decreasing. Hence we get

$$
E(1, A)<E(0, A)=F(A)=0 .
$$

Using Lemma 4.2 (ii), we find a constant $A_{*}(>A)$ such that $E(1, \lambda)<0$ for $\lambda \in\left[A, A_{*}\right]$, therefore $\lim _{r \rightarrow \infty} E(r, \lambda)<0$. The existence of $B_{*}$ is proved in the same way as above.

The next result is a direct consequence of Lemma 4.4 (ii) and Lemma 4.7.

Lemma 4.8. It holds that

$$
u(0) \geq A_{*} \text { for } u \in S^{+} \quad \text { and } \quad u(0) \leq-B_{*} \text { for } u \in S^{-} .
$$

We need the lemma below in Definition 4.11 later on.

Lemma 4.9. For any $\lambda>A$ (or $\lambda<-B$ ) there exists an $r>0$ such that $u(r, \lambda)=A$ (or $u(r, \lambda)=-B)$.

Proof. We first set $\varepsilon_{0}=\inf \{f(s): s \geq A\}$, which is positive because of (f2) and (f4). Let $\lambda>A$ and $u(r)$ denote $u(r, \lambda)$. We next write $C=\sup \left\{\left|u^{\prime}(r)\right|: r \geq\right.$ $0\}$ by Lemma 4.2. In contradiction to the assertion of the lemma, suppose that $u(r)>A$ for all $r \geq 0$. Then (4.1) implies

$$
u^{\prime \prime}=-\frac{n-1}{r} u^{\prime}-f(u) \leq \frac{n-1}{r} C-\varepsilon_{0} \leq-\frac{\varepsilon_{0}}{2} \quad \text { for } r \text { large enough, }
$$

which implies that $\lim _{r \rightarrow \infty} u^{\prime}(r)=-\infty$. This contradicts the boundedness of $u^{\prime}(r)$ and the proof is complete. 
Definition 4.10. Let $A_{*}$ and $B_{*}$ be determined by Lemma 4.7. We define positive constants $A_{1}, B_{1}, \theta_{0}$ and $\theta$ in the following way. First, we fix $A_{1}$ and $B_{1}$ such that $-B_{*}<-B_{1}<-B$ and $A<A_{1}<A_{*}$. Secondly, the constant $\theta_{0} \in(0,1)$ is determined by Lemma 3.2. Lastly, we define the constant $\theta \in(0,1)$ such that $\max \left(A_{1} / A_{*}, B_{1} / B_{*}, \theta_{0}\right)<\theta<1$. Therefore the conclusions of Lemmas 3.2 and 3.3 hold.

We recall that equation (4.1) has a singularity at $r=0$. To estimate the integral of $u^{\prime}(r)^{2} r^{n-1}$ over $[0, \infty)$, we divide the interval $[0, \infty)$ into a small neighborhood of $r=0$ and the other interval. To this end, we introduce the notation $t(\lambda)$. Then the interval $[0, t(\lambda))$ means the small neighborhood of $r=0$.

Definition 4.11. For any $\lambda \in\left(-\infty,-B_{*}\right] \cup\left[A_{*}, \infty\right)$ we define $t(\lambda)$ by

$$
t(\lambda)=\min \{r>0: u(r, \lambda)=\theta \lambda\}
$$

The set on the right-hand side is nonempty. Indeed, if $\lambda \geq A_{*}$, then the definition of $\theta$ implies that $\theta \lambda>A_{1}>A$, hence $u(0, \lambda)=\lambda>\theta \lambda>A$. Therefore Lemma 4.9 implies that the set is nonempty, and so $t(\lambda)$ is welldefined. From the definition of $t(\lambda)$ it follows that

$$
\begin{aligned}
& \lambda \geq u(r, \lambda) \geq \theta \lambda>A_{1} \quad \text { when } \quad \lambda \geq A_{*} \quad \text { and } \quad r \in[0, t(\lambda)], \\
& \lambda \leq u(r, \lambda) \leq \theta \lambda<-B_{1} \quad \text { when } \quad \lambda \leq-B_{*} \quad \text { and } \quad r \in[0, t(\lambda)] .
\end{aligned}
$$

In the two lemmas below, we present a few estimates for $t(\lambda)$, which will be useful for proving Lemma 4.14 later on and computing the integral of $u^{\prime}(r)^{2} r^{n-1}$ over $[0, t(\lambda)]$.

Lemma 4.12. We replace $\theta$ by a constant slightly less than 1 , if necessary. Then it holds that $0<t(\lambda)<1$ for $\lambda \in\left(-\infty,-B_{*}\right] \cup\left[A_{*}, \infty\right)$.

Proof. We rewrite (4.1) as

$$
\left(r^{n-1} u^{\prime}\right)^{\prime}=-r^{n-1} f(u)
$$

Integrating both sides twice, we have

$$
u(0)-u(r)=\int_{0}^{r} \frac{1}{s^{n-1}} \int_{0}^{s} f(u(\tau)) \tau^{n-1} d \tau d s .
$$

Let $u=u(r, \lambda)$ and substitute $r=t(\lambda)$. Then we get

$$
(1-\theta) \lambda=\int_{0}^{t(\lambda)} \frac{1}{s^{n-1}} \int_{0}^{s} f(u) \tau^{n-1} d \tau d s
$$

On the other hand, by assumption (f4) we have a constant $R>0$ such that $f(s) \geq 2 n s$ for $s \geq R$. Let $\lambda \geq A_{*}$ and $\theta \in(1 / 2,1)$. If $\lambda \geq 2 R$, then (4.13) implies that $R \leq \theta \lambda \leq u(r) \leq \lambda$ for $r \in(0, t(\lambda))$, and so (4.15) yields

$$
(1-\theta) \lambda \geq 2 n \int_{0}^{t(\lambda)} \frac{1}{s^{n-1}} \int_{0}^{s} u(\tau) \tau^{n-1} d \tau d s \geq \theta \lambda t(\lambda)^{2},
$$

hence,

$$
t(\lambda)^{2} \leq(1-\theta) / \theta<1
$$


If $A_{*} \leq \lambda \leq 2 R$, then $A_{1} \leq \theta \lambda \leq u(r) \leq 2 R$ for $r \in(0, t(\lambda))$. We now set $f_{0}=\min \left\{f(s): A_{1} \leq s \leq 2 R\right\}>0$, and hence (4.15) implies

$$
2(1-\theta) R \geq f_{0} \int_{0}^{t(\lambda)} \frac{1}{s^{n-1}} \int_{0}^{s} \tau^{n-1} d \tau d s=\frac{f_{0}}{2 n} t(\lambda)^{2} .
$$

Consequently, choosing $\theta$ to be sufficiently closed to 1 , we have $t(\lambda)<1$. For $\lambda \leq-B_{*}$ also, our method remains valid.

From now on, we always fix $\theta$ for which the conclusion of Lemma 4.12 is valid. In the next lemma, we estimate $t(\lambda)$ from below.

Lemma 4.13. There exists a positive constant $C$ such that

$$
C t(\lambda) \geq|\lambda| F(\lambda)^{-1 / 2} \quad \text { for } \lambda \in\left(-\infty,-B_{*}\right] \cup\left[A_{*}, \infty\right) .
$$

Proof. Let $\lambda \geq A_{*}$ and $u(r)=u(r, \lambda)$. By (3.4) there exists a constant $C_{1}>0$ such that $s f(s) \leq C_{1} F(s)$ for $s \in\left[A_{1}, \infty\right)$. We note that $A_{1} \leq \theta \lambda \leq u(r) \leq \lambda$ for $r \in(0, t(\lambda))$ by (4.13). Then the right-hand side of (4.15) is estimated as

$$
\int_{0}^{t(\lambda)} \frac{1}{s^{n-1}} \int_{0}^{s} f(u) \tau^{n-1} d \tau d s \leq C_{1} \int_{0}^{t(\lambda)} \frac{1}{s^{n-1}} \int_{0}^{s} \frac{F(u)}{u} \tau^{n-1} d \tau d s \leq \frac{C_{1} F(\lambda)}{2 n \theta \lambda} t(\lambda)^{2} .
$$

This inequality together with (4.15) implies the desired conclusion. In case of $\lambda \leq-B_{*}$ the above argument is also valid and the proof is complete.

We prove that the energy diverges to $+\infty$ as $\lambda \rightarrow \pm \infty$, which will be used in Section 6.

Lemma 4.14. We have

$$
\lim _{\lambda \rightarrow \pm \infty} E(R, \lambda)=\infty \quad \text { for any } \quad R \geq 0 .
$$

Proof. Since $E(r)$ is decreasing, it is sufficient to prove the lemma for $R \geq 1$. Let $R \geq 1$. We fix $\lambda \in \mathbf{R}$ arbitrarily and write $u(r)=u(r, \lambda)$ and $E(r)=$ $E(r, \lambda)$. The lemma is proved by using Pohozaev's identity [9]:

$$
2 E(R) R^{n}+(n-2) u^{\prime}(R) u(R) R^{n-1}=\int_{0}^{R}(2 n F(u)-(n-2) u f(u)) r^{n-1} d r \text {. }
$$

This relation is obtained in the following way. First, multiplying (4.1) by $u(r) r^{n-1}$ and integrating the resultant identity over $[0, R]$, we obtain

$$
\int_{0}^{R}\left|u^{\prime}\right|^{2} r^{n-1} d r=\int_{0}^{R} u f(u) r^{n-1} d r+u^{\prime}(R) u(R) R^{n-1} .
$$

Next, multiplying (4.1) by $u^{\prime}(r) r^{n}$ and integrating it over $[0, R]$, we have

$$
\frac{n-2}{2} \int_{0}^{R}\left|u^{\prime}\right|^{2} r^{n-1} d r+E(R) R^{n}=n \int_{0}^{R} F(u) r^{n-1} d r .
$$

Lastly, combining (4.17) and (4.18), we obtain (4.16).

On the other hand, there exist constants $\nu>0$ and $C>0$ such that

$$
2 n F(s)-(n-2) s f(s) \geq \nu F(s)-C \text { for } s \in \mathbf{R} \text {. }
$$

Indeed, if $n=2$, we choose $\nu=2 n=4$ to get (4.19). If $n \geq 3$, we have a small constant $\nu>0$ by (f5) such that 


$$
\frac{s f(s)}{F(s)} \leq \frac{2 n-\nu}{n-2} \quad \text { for }|s| \text { large enough, }
$$

which implies (4.19).

Since $\lim _{s \rightarrow \pm \infty} f(s) / s=\infty$, one finds a constant $C_{0}>0$ such that

$$
s^{2} / 2 \leq F(s)+C_{0} \quad \text { for } s \in \mathbf{R} \text {. }
$$

Hence we have

$$
u^{\prime}(R) u(R) \leq \frac{1}{2} u^{\prime}(R)^{2}+\frac{1}{2} u(R)^{2} \leq E(R)+C_{0} .
$$

Substituting (4.19) and (4.21) into (4.16), we obtain

$$
C_{1} E(R)+C_{2} \geq \int_{0}^{R} F(u) r^{n-1} d r,
$$

where $C_{1}$ and $C_{2}$ are positive constants depending only on $R$. We will estimate the right-hand side. Let $t=t(\lambda)$ be defined by Definition 4.11. From (4.20) it follows that $F(s)+C_{0} \geq 0$ for $s \in \mathbf{R}$. Adding $\left(C_{0} / n\right) R^{n}$ to both sides of (4.22), we have

$$
C_{1} E(R)+C_{3} \geq \int_{0}^{R}\left(F(u)+C_{0}\right) r^{n-1} d r,
$$

where $C_{3}=C_{2}+\left(C_{0} / n\right) R^{n}$. Note that the integrand on the right-hand side is nonnegative and that $t(\lambda) \leq 1 \leq R$ by Lemma 4.12. Therefore we have

$$
\int_{0}^{R}\left(F(u)+C_{0}\right) r^{n-1} d r \geq \int_{0}^{t(\lambda)} F(u) r^{n-1} d r .
$$

Let $\lambda \geq A_{*}$. By (4.13) we have that $A_{1} \leq \theta \lambda \leq u(r) \leq \lambda$ and $F(\theta \lambda) \leq F(u(r))$ for $r \in(0, t(\lambda))$. Hence Lemma 3.2 with $s=\lambda$ implies that

$$
\int_{0}^{t(\lambda)} F(u) r^{n-1} d r \geq \int_{0}^{t(\lambda)} F(\theta \lambda) r^{n-1} d r \geq \frac{1}{2 n} F(\lambda) t(\lambda)^{n} .
$$

We use Lemmas 4.13 and 3.3 to estimate the right-hand side as

$$
(2 n)^{-1} F(\lambda) t(\lambda)^{n} \geq C_{4} F(\lambda)^{-(n-2) / 2} \lambda^{n} \geq C_{5} \lambda^{n-(p+1)(n-2) / 2} .
$$

Combining (4.23), (4.24), (4.25) and (4.26), we obtain

$$
C_{1} E(R)+C_{3} \geq C_{5} \lambda^{n-(p+1)(n-2) / 2} \longrightarrow \infty \quad \text { as } \lambda \rightarrow \infty \text {. }
$$

Here we have used that $n-(p+1)(n-2) / 2>0$ by Lemma 3.3. The argument above remains valid for the case where $\lambda \leq-B_{*}$. Thus the proof is complete.

Recall the fact that $E(r)>0$ for $r \geq 0$ and $u \in S$. Then we have an a priori estimate connected with $\left|u^{\prime}(r)\right|$ and $|u(r)|$.

Lemma 4.15. There exist positive constants $a_{0}$ and $C$ such that

$$
C\left|u^{\prime}(r)\right| \geq|u(r)|^{(q+1) / 2}
$$

provided that $|u(r)| \leq a_{0}$ and $u \in S$. Here $q$ is the constant in (f3).

Proof. By (f3) there exist positive constants $m$ and $a_{0}$ such that $0<a_{0}<$ $\min (a, b)$ and $F(s) \leq-m|s|^{q+1}$ for $|s| \leq a_{0}$. Let $u \in S$ and $r \geq 0$ be a point satisfying $|u(r)| \leq a_{0}$. Since $E(r)=\left|u^{\prime}\right|^{2} / 2+F(u)>0$ by Lemma 4.4 (ii), we have that $\left|u^{\prime}\right|^{2} / 2-m|u|^{q+1}>0$, which implies (4.27). 


\section{Proof of the Lower estimate}

In this section we prove the proposition below.

Proposition 5.1. There exists a constant $C>0$ such that

$$
C(k+1)^{n} \leq \int_{0}^{\infty}\left|u^{\prime}\right|^{2} r^{n-1} d r \quad \text { for } u \in S_{k}
$$

The proof of Proposition 5.1 is based on a nonlinear variant of Prüfer transformation. See [3, p. 332] for the usual linear Prüfer transformation. Our goal is to find the relation between the $L^{2}$-norm of $\nabla u$ for a solution $u$ and the number of zeros of $u$. The former is a continuous quantity, but the latter is a discrete one. To connect these quantities, we rewrite equation (2.1) to the first order system

$$
\left\{\begin{array}{l}
u^{\prime}=v, \\
v^{\prime}=-\frac{n-1}{r} v-f(u) .
\end{array}\right.
$$

Then the solution $(u(r), v(r))$ draws a smooth curve which does not contain the origin in the $(u, v)$-phase plain. Here we choose $v$-axis and $u$-axis as axes of abscissa and ordinate, respectively. Then we will prove later on that if $u(\tau)=0$ at some $\tau$, then the orbit $(u(r), v(r))$ intersects the $v$-axis at $\tau$ and moves counterclockwise around the origin as $r$ increases in a small neighborhood of $\tau$. Therefore, the number of zeros can be represented by the winding number of the orbit as $r$ varies over the interval $[0, \infty)$. To calculate the winding number, we introduce polar coordinates and define the argument $\varphi$ as below.

Definition 5.2. For $u \in S$ we define the functions $\rho(r)$ and $\varphi(r)$ by

$$
\begin{aligned}
u^{\prime} & =\rho \cos \varphi, \\
Y(u) & =\rho \sin \varphi, \\
\varphi(0) & =\frac{\pi}{2},
\end{aligned}
$$

where $Y(s)=\operatorname{sgn}(s) \sqrt{G(s)}$ and $G(s)$ is defined by Lemma 3.4.

Observing Lemma 3.4 and (3.9), we see that $s Y(s)>0$ for $s \neq 0$ and moreover that $Y \in C^{1}(\mathbf{R}), Y^{\prime}(s)=(1 / 2)|g(s)| G(s)^{-1 / 2}$ for $s \neq 0$ and $Y^{\prime}(0)=$ 0 . The relations (5.1), (5.2) and (5.3) determine the functions $\rho(r)$ and $\varphi(r)$ of class $C^{1}[0, \infty)$ uniquely. In fact, since any nontrivial solution $u \neq 0$ of (2.1)-(2.2) has only simple zeros, we get

$$
\rho(r)=\left(u^{\prime}(r)^{2}+Y(u)^{2}\right)^{1 / 2}=\left(u^{\prime}(r)^{2}+G(u)\right)^{1 / 2}>0 \text { for } r \in[0, \infty) .
$$

Therefore $\rho(r)$ and $\varphi(r)$ are well-defined. Since the left-hand sides of (5.1) and (5.2) are continuously differentiable, the functions $\rho(r)$ and $\varphi(r)$ are of class $C^{1}[0, \infty)$ as well.

The definitions (5.1) and (5.2) transform (2.1) into a system of differential equations for $(\rho, \varphi)$. In particular, $\varphi^{\prime}$ satisfies the following equation.

Lemma 5.3. For $u \in S$ we have

$$
\rho^{2} \varphi^{\prime}=Y^{\prime}(u)\left\{\left|u^{\prime}\right|^{2}+2 f(u) \frac{G(u)}{g(u)}\right\}+\frac{n-1}{r} u^{\prime} Y(u) .
$$


Proof. Differentiating (5.1) with respect to $r$, we have

$$
u^{\prime \prime}=\rho^{\prime} \cos \varphi-\rho \varphi^{\prime} \sin \varphi .
$$

Substituting this relation into $(2.1)$ yields

$$
\frac{n-1}{r} u^{\prime}+f(u)=-\rho^{\prime} \cos \varphi+\rho \varphi^{\prime} \sin \varphi .
$$

Next, we differentiate (5.2) to obtain

$$
Y^{\prime}(u) u^{\prime}=\rho^{\prime} \sin \varphi+\rho \varphi^{\prime} \cos \varphi .
$$

Multiplying (5.6) by (5.2) and (5.7) by (5.1), and then summing up these identities, we obtain

$$
\rho^{2} \varphi^{\prime}=Y^{\prime}(u)\left|u^{\prime}\right|^{2}+\frac{n-1}{r} u^{\prime} Y(u)+f(u) Y(u) .
$$

Substituting $Y(u)=2 Y^{\prime}(u) G(u) / g(u)$ into the last term on the above identity, we get the desired relation (5.5).

The next lemma implies that the orbit $\left(u^{\prime}, Y(u)\right)$, hence $\left(u^{\prime}, u\right)$ also, winds counterclockwise around the origin as $r$ increases in a small neighborhood of each zero of $u$.

Lemma 5.4. Let $u \in S$. Then for any zero $\tau$ of $u$ there is an $\varepsilon>0$ such that

$$
\varphi^{\prime}(r)>0 \quad \text { if } 0<|r-\tau|<\varepsilon .
$$

Therefore $\varphi(r)$ is strictly increasing in some neighborhoods of zeros of $u(r)$.

Proof. Since $u$ has only simple zeros, $u^{\prime}(\tau) \neq 0$ and $\tau \neq 0$. Substituting $Y(u)=2 Y^{\prime}(u) G(u) / g(u)$ into (5.5), we get

$$
\frac{\rho^{2}}{Y^{\prime}(u)} \varphi^{\prime}=\left|u^{\prime}\right|^{2}+\left\{2 f(u)+\frac{2(n-1)}{r} u^{\prime}\right\} \frac{G(u)}{g(u)} .
$$

From the definition (3.9) of $g(s)$ it follows that

$$
\frac{G(u(r))}{g(u(r))}=\frac{1}{\mu+1} u(r) \rightarrow 0 \quad \text { as } \quad r \rightarrow \tau,
$$

and so,

$$
\lim _{r \rightarrow \tau} \frac{\rho^{2}}{Y^{\prime}(u)} \varphi^{\prime}=u^{\prime}(\tau)^{2}>0 .
$$

We note that $Y^{\prime}(u)=(1 / 2)|g(u)| G(u)^{-1 / 2}>0$ for $u \neq 0$. Therefore, if $|r-\tau|>0$ is sufficiently small, then we obtain $\rho(r)^{2} \varphi^{\prime}(r)>0$. This completes the proof.

In the following lemma, we show that for any solution $u$, the value of $\varphi\left(R_{2}\right)-$ $\varphi\left(R_{1}\right)$ reflects the number of zeros of $u$ in $\left[R_{1}, R_{2}\right]$. Therefore we have only to calculate the value of $\varphi(r)$ instead of the number of zeros.

Lemma 5.5. (i) Let $u \in S_{k}$ and $\left\{r_{j}\right\}_{j=1}^{k}\left(0<r_{1}<r_{2}<\cdots<r_{k}\right)$ denote its zeros. Then we have

$$
\begin{array}{cl}
\varphi\left(r_{j}\right)=j \pi \quad \text { for } 1 \leq j \leq k, \\
(j-1) \pi<\varphi(r)<j \pi \quad \text { for } \quad r_{j-1}<r<r_{j}, \quad 1 \leq j \leq k+1,
\end{array}
$$

where we set $r_{0}=0$ and $r_{k+1}=\infty$. 
(ii) Let $u \not \equiv 0$ satisfy (2.1) and $u^{\prime}(0)=0$. Then it holds that $u \in S_{k}$ if and only if $\varphi(r)$ tends to $(k+1) \pi$ as $r \rightarrow \infty$.

Proof. Notice by (5.2) that $u(\tau)=0$ if and only if $\varphi(\tau)=0(\bmod \pi)$. Consider the first zero $r_{1}$ of $u(r)$. Then it holds that $u(r) \neq 0\left(0<r<r_{1}\right)$ and $u\left(r_{1}\right)=0$, in other words, $\varphi(r) \neq 0(\bmod \pi)$ for $0<r<r_{1}$ and $\varphi\left(r_{1}\right)=0(\bmod \pi)$. Since $\varphi(0)=\pi / 2$ by $(5.3)$, it follows that $0<\varphi(r)<\pi$ for $0<r<r_{1}$ and $\varphi\left(r_{1}\right)=0$ or $\pi$. Then Lemma 5.4 implies that $\varphi\left(r_{1}\right)=\pi$ and $\varphi(r)>\pi$ for $r$ slightly larger than $r_{1}$. Since $u(r) \neq 0$ for $r_{1}<r<r_{2}$, it holds that $\pi<\varphi(r)<2 \pi$ for $r_{1}<r<r_{2}$. Proceeding with this argument inductively, we obtain (i).

We prove assertion (ii). The definitions (5.1) and (5.2) imply

$$
\tan \varphi=\frac{Y(u)}{u^{\prime}} .
$$

Let $u \in S_{k}$. Then assertion (i) gives

$$
k \pi<\varphi(r)<(k+1) \pi \quad \text { for } \quad r \in\left(r_{k}, \infty\right) .
$$

Observing Lemma 4.6, we see that

$$
u(r) u^{\prime}(r)<0 \text { for } r \in\left(t_{k}, \infty\right),
$$

where $t_{k}$ denotes the largest zero of $u^{\prime}(r)$. In case of $k=0$, we set $r_{0}=0$ in (5.9) and $t_{0}=0$ in (5.10). Since $s Y(s)>0$ for $s \neq 0$, it follows from (5.8) and $(5.10)$ that

$$
\tan \varphi(r)<0 \quad \text { for } \quad r \in\left(t_{k}, \infty\right) .
$$

Combining (5.9) and (5.11), we get

$$
(k+1 / 2) \pi<\varphi(r)<(k+1) \pi \text { for } r \in\left(t_{k}, \infty\right) .
$$

On the other hand, there is a constant $C>0$ by (3.9) such that $G(u) \leq C|u|^{\mu+1}$ for $|u|$ sufficiently small. Then (5.8) together with Lemma 4.15 implies that

$$
|\tan \varphi|=\frac{\sqrt{G(u)}}{\left|u^{\prime}\right|} \leq C_{1}|u|^{(\mu-q) / 2} \text { for } r \text { large enough. }
$$

Since $\mu>2 q-1 \geq q$ by (3.7), $\tan \varphi(r)$ tends to 0 as $r \rightarrow \infty$. This together with (5.12) means

$$
\lim _{r \rightarrow \infty} \varphi(r)=(k+1) \pi .
$$

Conversely, suppose that $\varphi(r)$ satisfies (5.13). Then $\tan \varphi(r)$ tends to 0 as $r \rightarrow \infty$. Since $\left|u^{\prime}(r)\right|$ is bounded by Lemma 4.2, we have

$$
|Y(u)|=\left|u^{\prime} \tan \varphi\right| \longrightarrow 0 \quad \text { as } r \rightarrow \infty .
$$

This implies $\lim _{r \rightarrow \infty} u(r)=0$, i.e., $u \in S$. Then we have (5.10) for $r$ large enough, and hence $\tan \varphi(r)<0$ by (5.8). This fact with (5.13) implies that

$$
(k+1 / 2) \pi<\varphi(r)<(k+1) \pi \quad \text { for all } r \text { large enough. }
$$

Therefore assertion (i) proves $u \in S_{k}$. Thus the proof is complete.

We now explain our plan to prove Proposition 5.1 and Theorem 1. Using (5.4) and dividing (5.5) by $\rho^{2}$, we obtain

$$
\varphi^{\prime}=Z\left(u, u^{\prime}, r\right)
$$


where $Z$ is a function of variables $u, u^{\prime}$ and $r$, which can be written explicitly by (5.5). Integrating both sides over $[0, \infty)$, we have

$$
\varphi(\infty)-\varphi(0)=\int_{0}^{\infty} Z\left(u, u^{\prime}, r\right) d r
$$

The left-hand side represents the number of zeros of $u$ in $[0, \infty)$ by Lemma 5.5. The right-hand side will be estimated from above or below via the $L^{2}$-norm of $\nabla u$. More precisely, we will prove that

$$
C_{1}\|\nabla u\|_{L^{2}}^{2 / n} \leq \int_{0}^{\infty} Z\left(u, u^{\prime}, r\right) d r \leq C_{2}\|\nabla u\|_{L^{2}}^{2 / n} .
$$

To make a success of the computation above, we adopted the nonlinear transformation (5.2). Consequently, we can connect the number of zeros and the Sobolev norm by using (5.14) and (5.15).

In what follows, we prove (5.15). To do so, we alter (5.5) into a simpler form as below.

Lemma 5.6. There exist positive constants $C_{1}$ and $C_{2}$ such that

$$
\begin{aligned}
& \varphi^{\prime} \leq C_{1}(g(u) / u)^{1 / 2}+(n-1) \frac{u^{\prime} Y(u)}{r \rho^{2}}, \\
& \varphi^{\prime} \geq C_{2}(g(u) / u)^{1 / 2}+(n-1) \frac{u^{\prime} Y(u)}{r \rho^{2}} .
\end{aligned}
$$

Proof. From the definition (3.9) of $g(s)$ together with (f5), there exist constants $C_{1}, C_{2}>0$ such that

$$
C_{1} s g(s) \leq G(s) \leq C_{2} s g(s) \quad \text { for } \quad s \in \mathbf{R} .
$$

Using this inequality in the relation $Y^{\prime}(s)=(1 / 2)|g(s)| G(s)^{-1 / 2}$, we have

$$
C_{3}(g(s) / s)^{1 / 2} \leq Y^{\prime}(s) \leq C_{4}(g(s) / s)^{1 / 2} \quad \text { for some } C_{3}, C_{4}>0 .
$$

On the other hand, by Lemma 3.4 (iii) there exists a positive constant $C$ such that $f(s) / g(s) \leq C$ for all $s \in \mathbf{R}$. Then we have

$$
\left|u^{\prime}\right|^{2}+2 f(u) \frac{G(u)}{g(u)} \leq\left|u^{\prime}\right|^{2}+2 C G(u) \leq \max (1,2 C) \rho^{2},
$$

where we have used the relation $\rho^{2}=\left|u^{\prime}\right|^{2}+G(u)$ by (5.4). Combining (5.18), (5.19) and (5.5), we obtain (5.16).

We prove (5.17). Using $f(u)=g(u)-h(u)$ and Lemma 3.4 (iv), we get

$$
\left|u^{\prime}\right|^{2}+2 f(u) \frac{G(u)}{g(u)}=\left|u^{\prime}\right|^{2}+2 G(u)-2 h(u) \frac{G(u)}{g(u)} \geq\left|u^{\prime}\right|^{2}+2 G(u)-H(u) .
$$

Now, recall Lemma 4.4 (ii), that is,

$$
E(r) \equiv \frac{1}{2}\left|u^{\prime}\right|^{2}+G(u)-H(u)>0 .
$$

Then the two inequalities above yield

$$
\left|u^{\prime}\right|^{2}+2 f(u) \frac{G(u)}{g(u)} \geq \frac{1}{2}\left|u^{\prime}\right|^{2}+G(u) \geq \frac{1}{2} \rho^{2} .
$$

By (5.18), (5.20) and (5.5) we have (5.17). The proof is complete. 
We integrate $(5.16)$ over $[0, \infty)$ and estimate the integral of the first term on the right-hand side. To this end, we divide the interval $[0, \infty)$ into two domains $I$ and $J$ in the following way.

Definition 5.7. Let $u \in S$. Let $a_{0}$ be defined by Lemma 4.15. For $\delta \in\left(0, a_{0}\right)$ we set

$$
\begin{aligned}
& I=I(\delta, u)=\{r \in(0, \infty):|u(r)| \geq \delta\}, \\
& J=J(\delta, u)=\{r \in(0, \infty):|u(r)|<\delta\} .
\end{aligned}
$$

Lemma 5.8. For any $\nu>(q-1) / 2$ there exists a positive constant $C_{\nu}$ such that

$$
\int_{J}|u|^{\nu} d r \leq C_{\nu} \delta^{(2 \nu-q+1) / 2}(k+1) \quad \text { for } u \in S_{k} \text { and } \delta \in\left(0, a_{0}\right) \text {. }
$$

Proof. Let $u \in S_{k}$ and $\left\{r_{i}\right\}_{i=1}^{k}\left(0<r_{1}<r_{2}<\cdots<r_{k}\right)$ denote the zeros of $u$. Recalling the outline of the graph of $u(r)$, that is, noting Lemma 4.6, we see that $J$ is represented as

where

$$
J=\bigcup_{i=1}^{k+1}\left(\alpha_{i}, \beta_{i}\right),
$$

$$
\begin{gathered}
\alpha_{i}<r_{i}<\beta_{i} \quad(1 \leq i \leq k), \quad \alpha_{k+1}<\beta_{k+1}=\infty, \\
\left|u\left(\alpha_{i}\right)\right|=\left|u\left(\beta_{i}\right)\right|=\delta, \quad u\left(\alpha_{i}\right) u\left(\beta_{i}\right)<0 \quad(1 \leq i \leq k), \\
\left|u\left(\alpha_{k+1}\right)\right|=\delta, \quad \text { and } \quad u\left(\beta_{k+1}\right)=u(\infty)=0 .
\end{gathered}
$$

We consider the interval $\left(\alpha_{i}, r_{i}\right)$ with $i \leq k$ in which $u(r)>0$ and $u^{\prime}(r)<0$. Hence $u\left(\alpha_{i}\right)=\delta$ and $u\left(r_{i}\right)=0$. Let $\sigma>0$. Then Lemma 4.15 gives

$$
-\left(u^{\sigma}\right)^{\prime}=-\sigma u^{\sigma-1} u^{\prime}=\sigma|u|^{\sigma-1}\left|u^{\prime}\right| \geq C_{\sigma}|u|^{(2 \sigma+q-1) / 2}
$$

for some $C_{\sigma}>0$. We integrate both sides over $\left[\alpha_{i}, r_{i}\right]$ to obtain

$$
C_{\sigma} \int_{\alpha_{i}}^{r_{i}}|u|^{(2 \sigma+q-1) / 2} d r \leq-u\left(r_{i}\right)^{\sigma}+u\left(\alpha_{i}\right)^{\sigma}=\delta^{\sigma} .
$$

In the case where $u(r)<0$ for $r \in\left(\alpha_{i}, r_{i}\right)$, moreover, in the interval $\left(r_{i}, \beta_{i}\right)$ or $\left(\alpha_{k+1}, \beta_{k+1}\right)$ even, the above estimate remains still valid. Summing up these inequalities on $i$ from 1 to $k+1$, we have

$$
C_{\sigma} \int_{J}|u|^{(2 \sigma+q-1) / 2} d r \leq(2 k+1) \delta^{\sigma} .
$$

Substituting $\sigma=(2 \nu-q+1) / 2>0$ into this inequality, we obtain the conclusion of Lemma 5.8.

By (3.9) there exists a constant $C>0$ such that

$$
(g(s) / s)^{1 / 2} \leq C|s|^{(\mu-1) / 2} \text { for }|s| \text { sufficiently small. }
$$

Using this inequality in Lemma 5.8, we have the following lemma.

Lemma 5.9. There exists a positive constant $C$ independent of $\delta$ and $k$ such that

$$
\int_{J}(g(u) / u)^{1 / 2} d r \leq C \delta^{(\mu-q) / 2}(k+1) \quad \text { for } u \in S_{k} \text { and } \delta \in\left(0, a_{0}\right) .
$$

The above lemma gives the estimate for the integral over $J$. Next we need to estimate the integral over $I(\delta, u)$ of $(g(u) / u)^{1 / 2}$. To this end, we present a brief lemma on the Lebesgue integral of nonnegative and monotone functions. 
Lemma 5.10. Let $f$ be a nonnegative function defined on $(0, \infty)$. Suppose that $I$ is a measurable subset of $(0, \infty)$. Then the following assertions (i) and (ii) hold:

(i) If $f$ is nondecreasing, then we have

$$
\int_{0}^{|I|} f(r) d r \leq \int_{I} f(r) d r
$$

(ii) If $f$ is nonincreasing, then we have

$$
\int_{I} f(r) d r \leq \int_{0}^{|I|} f(r) d r
$$

Here $|I|$ denotes the measure of $I$. Some of these integrals may be $+\infty$. But this lemma asserts that when the right-hand side is finite, so is the left-hand side, and hence the inequality is valid.

Proof. We prove (i) only, but the assertion (ii) can be treated in the same way. If $f(r)=0$ a.e. on $(0, \infty)$, then the inequality is trivial. Suppose that there exists a $\delta>0$ such that $f(r)>\delta$ for $r$ large enough since $f$ is nondecreasing and nonnegative. If $|I|=\infty$, both sides of the inequality (i) are equal to $+\infty$. Suppose that $|I|<\infty$. We first deal with the case where $I$ is a bounded open set. Hence it is represented as $I=\bigcup_{i=1}^{\infty}\left(a_{i}, b_{i}\right)$. We set $I_{k}=\bigcup_{i=1}^{k}\left(a_{i}, b_{i}\right)$. Rearranging it, we have $I_{k}=\bigcup_{i=1}^{k}\left(\alpha_{i}, \beta_{i}\right)$ where $0 \leq \alpha_{1}<\beta_{1} \leq \alpha_{2}<\cdots<$ $\beta_{k}<\infty$. Set $T_{j}=\sum_{i=1}^{j}\left(\beta_{i}-\alpha_{i}\right)$ for $1 \leq j \leq k$ and $T_{0}=0$. Then since $f$ is nondecreasing, we have

$$
\int_{\alpha_{i}}^{\beta_{i}} f(r) d r=\int_{T_{i-1}}^{T_{i}} f\left(r+\alpha_{i}-T_{i-1}\right) d r \geq \int_{T_{i-1}}^{T_{i}} f(r) d r .
$$

The summation on $i$ gives

$$
\int_{I_{k}} f(r) d r \geq \int_{0}^{T_{k}} f(r) d r=\int_{0}^{\left|I_{k}\right|} f(r) d r
$$

Since $I_{k} \subset I$ and $f \geq 0$, we get

$$
\int_{I} f(r) d r \geq \int_{0}^{\left|I_{k}\right|} f(r) d r
$$

Letting $k \rightarrow \infty$, we obtain (i). If $I$ is an unbounded open set, then we use an approximate sequence of bounded open sets to get the conclusion.

Let $I$ be a measurable set. Then there exists a sequence $\left\{I_{k}\right\}_{k=1}^{\infty}$ of open sets such that $I \subset I_{k}$ and $\lim _{k \rightarrow \infty}\left|I_{k}-I\right|=0$. Therefore we have

$$
\int_{I_{k}} f(r) d r \geq \int_{0}^{\left|I_{k}\right|} f(r) d r \geq \int_{0}^{|I|} f(r) d r .
$$

Letting $k \rightarrow \infty$, we obtain assertion (i) and the proof is complete.

Applying the above lemma, we can estimate the integral of $(g(u) / u)^{1 / 2}$ over $I(\delta, u)$.

Lemma 5.11. For any $\delta \in\left(0, a_{0}\right)$ there exists a constant $C_{\delta}$ depending on $\delta$ such that

$$
\int_{I}(g(u) / u)^{1 / 2} d r \leq C_{\delta}\left(\int_{I} u g(u) r^{n-1} d r\right)^{1 / n}
$$

for $u \in S$ and $I=I(\delta, u)$. 
Proof. Let $\delta \in\left(0, a_{0}\right)$. Then there exist constants $m(>n)$ and $C_{\delta}(>0)$ such that

$$
(g(s) / s)^{m / 2} \leq C_{\delta} s g(s) \quad \text { for }|s| \geq \delta .
$$

Indeed, by Lemma 3.3 and Lemma 3.4 (i) we have a constant $m(>n)$ such that

$$
\begin{gathered}
p+1<\frac{2 m}{m-2}<\infty \quad \text { if } n=2, \quad p+1<\frac{2 m}{m-2}<\frac{2 n}{n-2} \text { if } n \geq 3, \\
s g(s) \leq C|s|^{2 m /(m-2)} \quad \text { for } \quad s \in\left(-\infty,-B_{1}\right] \cup\left[A_{1}, \infty\right) .
\end{gathered}
$$

The last inequality remains still valid for $|s| \geq \delta$ if we replace $C$ by a larger constant $C_{\delta}$. Then the above inequality is equivalent to (5.22).

We use Hölder's inequality to get

$$
\begin{aligned}
\int_{I}(g(u) / u)^{1 / 2} d r & =\int_{I}(g(u) / u)^{1 / 2} r^{(n-1) / m} r^{-(n-1) / m} d r \\
& \leq\left(\int_{I}(g(u) / u)^{m / 2} r^{n-1} d r\right)^{1 / m}\left(\int_{I} r^{-m^{\prime}(n-1) / m} d r\right)^{1 / m^{\prime}},
\end{aligned}
$$

where $1 / m+1 / m^{\prime}=1$. By Lemma 5.10 (ii) the last term is estimated as

$$
\begin{aligned}
& \int_{I} r^{-m^{\prime}(n-1) / m} d r=\int_{I} r^{-(n-1) /(m-1)} d r \\
& \quad \leq \int_{0}^{|I|} r^{-(n-1) /(m-1)} d r=\frac{m-1}{m-n}|I|^{(m-n) /(m-1)} .
\end{aligned}
$$

Here we note that $|I|<\infty$. Indeed, $I$ is a bounded set because $u(r)$ tends to 0 as $r \rightarrow \infty$. Combining (5.22), (5.23) and (5.24), we obtain

$$
\int_{I}(g(u) / u)^{1 / 2} d r \leq C_{\delta}\left(\int_{I} u g(u) r^{n-1} d r\right)^{1 / m}|I|^{(m-n) / m} .
$$

On the other hand, the application of Lemma 5.10 (i) yields

$$
\int_{I} u g(u) r^{n-1} d r \geq D_{\delta} \int_{I} r^{n-1} d r \geq D_{\delta} \int_{0}^{|I|} r^{n-1} d r=\left(D_{\delta} / n\right)|I|^{n},
$$

where $D_{\delta}$ denotes the infimum of $s g(s)$ for $|s| \geq \delta$. The above inequality is rewritten as

$$
|I| \leq D_{\delta}^{\prime}\left(\int_{I} u g(u) r^{n-1} d r\right)^{1 / n}
$$

By (5.25) and (5.26) we obtain the conclusion (5.21) and the proof is complete.

Summing up the inequalities in the assertions of Lemmas 5.9 and 5.11, we get the lemma below.

Lemma 5.12. There exist constants $C, C_{\delta}>0$ such that

$$
\int_{0}^{\infty}(g(u) / u)^{1 / 2} d r \leq C_{\delta}\left(\int_{0}^{\infty} u g(u) r^{n-1} d r\right)^{1 / n}+C \delta^{(\mu-q) / 2}(k+1)
$$


for $u \in S_{k}$ and $\delta \in\left(0, a_{0}\right)$. Here $C_{\delta}$ depends only on $\delta$, but $C$ does not depend on anything.

Recall Definition 4.11. We modify the notation $t(\lambda)$ slightly, which has been defined for the initial data $\lambda$. But, in what follows we define $t(u)$ for any solution $u(\not \equiv 0)$ of $(2.1)$ with (2.2).

Definition 5.13. Observing Lemma 4.8 and Definition 4.11, we define $t(u)$ by

$$
t(u)=\min \{r>0: u(r)=\theta u(0)\} \quad \text { for } \quad u \in S .
$$

We now proceed to estimate the integral of the second term on the righthand side of $(5.16)$. The interval $[0, \infty)$ of integration is divided into three subintervals $[0, t(u)),[t(u), R)$ and $[R, \infty)$. Here $R$ will be determined later on. First, in the interval $[0, t(u))$ the function $u^{\prime} Y(u) / r \rho^{2}$ is negative. Indeed, Lemma 4.6 implies that $u^{\prime}(r) u(r)<0$ for $r \in(0, t(u))$ because $t(u)$ is less than the first zero $r_{1}$ of $u$. Since $s Y(s)>0$ for $s \neq 0$, it holds that $u^{\prime} Y(u)<0$ for $r \in(0, t(u))$. Next, we estimate the integral of $u^{\prime} Y(u) / r \rho^{2}$ over $[t(u), R)$.

Lemma 5.14. For any $\varepsilon>0$ and any $R \geq 1$ there exists a constant $C_{R, \varepsilon}>0$ depending only on $\varepsilon$ and $R$ such that

$$
\int_{t(u)}^{R} \frac{\left|u^{\prime} Y(u)\right|}{r \rho^{2}} d r \leq C_{R, \varepsilon}\left(\int_{0}^{t(u)} F(u(r)) r^{n-1} d r\right)^{\varepsilon}
$$

for any $u \in S$.

Proof. Let $u \in S^{+}$. For simplicity, we use the notations $\lambda=u(0)$ and $t=t(u)$. We recall (4.25), i.e.,

$$
\int_{0}^{t} F(u) r^{n-1} d r \geq \frac{1}{2 n} F(\lambda) t^{n}
$$

On the other hand, from (5.4) it follows that

$$
\frac{\left|u^{\prime} Y(u)\right|}{\rho^{2}} \leq \frac{\left|u^{\prime}\right|^{2}+Y(u)^{2}}{2 \rho^{2}} \leq \frac{1}{2},
$$

and therefore

$$
\int_{t}^{R} \frac{\left|u^{\prime} Y(u)\right|}{r \rho^{2}} d r \leq \frac{1}{2} \int_{t}^{R} r^{-1} d r \leq \frac{1}{2} t^{-\varepsilon} \int_{t}^{R} r^{-1+\varepsilon} d r \leq \frac{1}{2 \varepsilon} R^{\varepsilon} t^{-\varepsilon} .
$$

We now show that there exist constants $\alpha>0$ and $C_{0}>0$ such that

$$
C_{0} F(\lambda) t^{n} \geq t^{-\alpha} \text {. }
$$

From Lemmas 4.13 and 3.3, it follows that

$$
F(\lambda) t^{n+\alpha} \geq C_{1} \lambda^{n+\alpha} F(\lambda)^{-(n+\alpha-2) / 2} \geq C_{2} \lambda^{n+\alpha-(p+1)(n+\alpha-2) / 2},
$$

where $C_{1}, C_{2}>0$ are independent of $\lambda$. We fix $\alpha$ so small that

$$
2(p+1) /(p-1)-n>\alpha>0,
$$


and therefore $n+\alpha-(p+1)(n+\alpha-2) / 2>0$. Then we obtain the inequality (5.29) by using $\lambda \geq A_{*}$. Combining (5.28), (5.29) and (5.27), we obtain

$$
\begin{aligned}
\int_{t}^{R} \frac{\left|u^{\prime} Y(u)\right|}{r \rho^{2}} d r & \leq \frac{1}{2 \varepsilon} R^{\varepsilon} t^{-\varepsilon}=C_{1}\left(t^{-\alpha}\right)^{\varepsilon / \alpha} \\
& \leq C_{1}\left(C_{0} F(\lambda) t^{n}\right)^{\varepsilon / \alpha} \leq C_{2}\left(\int_{0}^{t} F(u) r^{n-1} d r\right)^{\varepsilon / \alpha},
\end{aligned}
$$

where each $C_{i}(0 \leq i \leq 2)$ depends only on $R, \alpha$ and $\varepsilon$. Since $\varepsilon$ is an arbitrary positive number and $\alpha$ is a positive constant, we can replace $\varepsilon / \alpha$ by $\varepsilon$. This completes the proof.

The lemma above gives the estimate for the integral over $[t(u), R)$. In the next lemma, we estimate the integral over $\left[R, R_{1}\right)$ or $[R, \infty)$ of $\left|u^{\prime} Y(u)\right| / r \rho^{2}$.

Lemma 5.15. For any $\sigma>0$ there exist constants $C, C_{\sigma}>0$ such that

$$
\int_{R}^{R_{1}} \frac{\left|u^{\prime} Y(u)\right|}{r \rho^{2}} d r \leq \frac{C_{\sigma}}{R} \int_{R}^{R_{1}}(g(u) / u)^{1 / 2} d r+C \sigma^{(\mu-2 q+1) / 2}(k+1)
$$

for any $1 \leq R<R_{1} \leq \infty$ and $u \in S_{k}$. Here $C_{\sigma}$ depends only on $\sigma$, but $C$ does not on anything.

Proof. Since $g(s) / s>0$ for $s \neq 0$ and $\lim _{s \rightarrow \pm \infty} g(s) / s=\infty$, we set

$$
C_{\sigma}=\sup \left\{(1 / 2)(s / g(s))^{1 / 2}:|s| \geq \sigma\right\} .
$$

It suffices to prove the lemma for $\sigma \in\left(0, a_{0}\right)$ only. We use the notations $I=I(\sigma, u)$ and $J=J(\sigma, u)$ defined by Definition 5.7 with $\delta=\sigma$. Then for $r \in\left[R, R_{1}\right) \cap I(\sigma, u)$ it holds that

$$
\frac{\left|u^{\prime} Y(u)\right|}{r \rho^{2}} \leq \frac{\left|u^{\prime}\right|^{2}+Y(u)^{2}}{2 r \rho^{2}} \leq \frac{1}{2 R} \leq \frac{C_{\sigma}}{R}(g(u) / u)^{1 / 2},
$$

and therefore

$$
\int_{\left[R, R_{1}\right) \cap I} \frac{\left|u^{\prime} Y(u)\right|}{r \rho^{2}} d r \leq \frac{C_{\sigma}}{R} \int_{R}^{R_{1}}(g(u) / u)^{1 / 2} d r .
$$

Next we use that $r \geq R \geq 1$ for $r \in\left[R, R_{1}\right)$ and employ the inequality that $G(u) \leq C|u|^{\mu+1}$ for $|u|$ small enough because of (3.9). Then Lemma 4.15 gives for $r \in\left[R, R_{1}\right) \cap J$,

$$
\frac{\left|u^{\prime} Y(u)\right|}{r \rho^{2}} \leq \frac{\left|u^{\prime}\right| G(u)^{1 / 2}}{\left|u^{\prime}\right|^{2}}=\frac{G(u)^{1 / 2}}{\left|u^{\prime}\right|} \leq C|u|^{(\mu-q) / 2} .
$$

Hence Lemma 5.8 with $J=J(\sigma, u)$ and $\nu=(\mu-q) / 2$ implies that

$$
\int_{\left[R, R_{1}\right) \cap J} \frac{\left|u^{\prime} Y(u)\right|}{r \rho^{2}} d r \leq C \int_{J}|u|^{(\mu-q) / 2} d r \leq C \sigma^{(\mu-2 q+1) / 2}(k+1) .
$$

Here we note that the assumption of Lemma 5.8 is fulfilled since $\nu=(\mu-q) / 2>$ $(q-1) / 2$ by (3.7). Summing up (5.30) and (5.31), we obtain the conclusion.

We connect the two integrals of $u g(u)$ and $u^{\prime}(r)^{2}$ as below. 
Lemma 5.16. There exists a constant $C>0$ such that

$$
\int_{0}^{\infty} u g(u) r^{n-1} d r \leq C \int_{0}^{\infty}\left|u^{\prime}\right|^{2} r^{n-1} d r \quad \text { for } u \in S .
$$

Proof. Let $u \in S$. We recall Lemma 4.4 (iv), i.e.,

$$
\sup _{0 \leq r<\infty}\left|u^{\prime}(r)\right| r^{n-1}<\infty
$$

Therefore, letting $R \rightarrow \infty$ in (4.17), we have

$$
\int_{0}^{\infty}\left|u^{\prime}\right|^{2} r^{n-1} d r=\int_{0}^{\infty} u f(u) r^{n-1} d r
$$

We now set

$$
K=\left\{r \in(0, \infty):-b_{1}<u(r)<a_{1}\right\} \text { and } L=(0, \infty) \backslash K,
$$

where $a_{1}$ and $b_{1}$ are the constants fixed in Lemma 3.4, and so $a_{1} \in(a, A)$ and $-b_{1} \in(-B,-b)$. Then there exists a $C>0$ independent of $u$ such that

$$
C \int_{0}^{\infty}\left|u^{\prime}\right|^{2} r^{n-1} d r \geq \int_{K}(-u f(u)+u g(u)) r^{n-1} d r .
$$

This will be proved soon. Summing up (5.33) and (5.34), we have

$$
(1+C) \int_{0}^{\infty}\left|u^{\prime}\right|^{2} r^{n-1} d r \geq \int_{L} u f(u) r^{n-1} d r+\int_{K} u g(u) r^{n-1} d r=\int_{0}^{\infty} u g(u) r^{n-1} d r,
$$

where we have used that $f(u)=g(u)$ for $r \in L$ by Lemma 3.4 (i). Therefore we get (5.32).

We show (5.34). To this end, it suffices to prove the stronger assertion

$$
C\left|u^{\prime}\right|^{2} \geq|u f(u)|+u g(u) \quad \text { for } \quad r \in K \text {. }
$$

Since $\lim \sup _{s \rightarrow 0} f(s) /|s|^{q-1} s<0$ and $\limsup _{s \rightarrow 0}|s f(s) / F(s)|<\infty$, there exist constants $\delta_{0}>0$ and $C_{i}>0(1 \leq i \leq 4)$ such that

$$
\begin{gathered}
|s f(s)| \leq C_{1}|F(s)|=-C_{1} F(s), \\
s g(s) \leq C_{2}|s|^{\mu+1} \leq C_{3}|s|^{q+1} \leq-C_{4} F(s)
\end{gathered}
$$

for $|s| \leq \delta_{0}$. We note that $-F(s)>0$ for $s \in\left[-b_{1},-\delta_{0}\right] \cup\left[\delta_{0}, a_{1}\right]$. Hence the inequalities (5.36) and (5.37) remain valid for $s \in\left[-b_{1}, a_{1}\right]$ if we replace each $C_{i}$ by a larger constant. We sum up (5.36) and (5.37) to get

$$
-C F(u) \geq|u f(u)|+u g(u) \text { for } r \in K,
$$

where $C$ is independent of $u$. Since $E(r)=\left|u^{\prime}\right|^{2} / 2+F(u)>0$ by Lemma 4.4 (ii), we have

$$
\frac{1}{2} u^{\prime}(r)^{2} \geq-F(u)
$$

By (5.38) and (5.39) we obtain (5.35) and the proof is complete.

We are now in a position to give the proof of Proposition 5.1.

Proof of Proposition 5.1. Let $u \in S_{k}$. We define $t=t(u)$ by Definition 5.13. Integrating (5.16) over $[0, \infty)$ and using Lemma 5.5, we have

$$
(k+1 / 2) \pi \leq C_{1} \int_{0}^{\infty}(g(u) / u)^{1 / 2} d r+(n-1) \int_{0}^{\infty} \frac{u^{\prime} Y(u)}{r \rho^{2}} d r .
$$


We estimate the second term on the right-hand side. In view of the outline of the graph of $u(r)$ (recall Lemma 4.6) we see that $u^{\prime}(r) u(r)<0$ for $0<r<t(u)$, and hence

$$
\int_{0}^{t} \frac{u^{\prime} Y(u)}{r \rho^{2}} d r<0 .
$$

Next, it follows from Lemmas 5.14 and 5.15 with $R=1$ and $R_{1}=\infty$ that (5.42)

$$
\begin{aligned}
\left(\int_{t}^{1}+\int_{1}^{\infty}\right) \frac{\left|u^{\prime} Y(u)\right|}{r \rho^{2}} d r & \leq C_{\varepsilon}\left(\int_{0}^{t} F(u) r^{n-1} d r\right)^{\varepsilon} \\
& +C_{\sigma} \int_{1}^{\infty}(g(u) / u)^{1 / 2} d r+C \sigma^{(\mu-2 q+1) / 2}(k+1) .
\end{aligned}
$$

Here and in what follows, $C_{\varepsilon}$ and $C_{\sigma}$ denote various positive constants depending on $\varepsilon$ and $\sigma$, respectively. However, $C, C_{1}$ and $C_{2}$ denote positive constants independent of $\varepsilon$ and $\delta$. We set $\varepsilon=1 / n$ and combine (5.40), (5.41) and (5.42) to get

$$
\begin{aligned}
\frac{1}{2}(k+1) \leq & k+\frac{1}{2} \leq C_{\sigma} \int_{0}^{\infty}(g(u) / u)^{\mathrm{i} / 2} d r . \\
& +C_{1}\left(\int_{0}^{t} F(u) r^{n-1} d r\right)^{1 / n}+C_{2} \sigma^{(\mu-2 q+1) / 2}(k+1) .
\end{aligned}
$$

We choose $\sigma>0$ so small that $C_{2} \sigma^{(\mu-2 q+1) / 2}<1 / 4$ to get

$$
\frac{1}{4}(k+1) \leq C_{1} \int_{0}^{\infty}(g(u) / u)^{1 / 2} d r+C_{2}\left(\int_{0}^{t} F(u) r^{n-1} d r\right)^{1 / n} .
$$

Using Lemma 5.12, we have

$$
\begin{aligned}
\frac{1}{4}(k+1) \leq & C_{\delta}\left(\int_{0}^{\infty} u g(u) r^{n-1} d r\right)^{1 / n}+C_{1} \delta^{(\mu-q) / 2}(k+1) \\
& +C_{2}\left(\int_{0}^{t} F(u) r^{n-1} d r\right)^{1 / n} .
\end{aligned}
$$

Fix $\delta>0$ so small that $C_{1} \delta^{(\mu-q) / 2}<1 / 8$. Then it follows that

$$
((k+1) / 8)^{n} \leq C_{1} \int_{0}^{\infty} u g(u) r^{n-1} d r+C_{2} \int_{0}^{t} F(u) r^{n-1} d r .
$$

We here recall Lemma 4.8, (4.13) and (4.14). Then it holds that

$$
\begin{array}{lll}
u(r) \geq A_{1} & \text { when } \quad r \in[0, t(u)] \text { and } \quad u \in S^{+}, \\
u(r) \leq-B_{1} & \text { when } \quad r \in[0, t(u)] \text { and } u \in S^{-} .
\end{array}
$$

Moreover, by (f5) and Lemma 3.4 there exists a constant $C>0$ such that

$$
F(s) \leq C s f(s)=\operatorname{Cs} g(s) \quad \text { for } \quad s \in\left(-\infty,-B_{1}\right] \cup\left[A_{1}, \infty\right),
$$

and so

$$
F(u(r)) \leq C u(r) g(u(r)) \quad \text { for } \quad r \in[0, t] \text { and } u \in S .
$$


Therefore it follows from (5.43) that

$$
(k+1)^{n} \leq C \int_{0}^{\infty} u g(u) r^{n-1} d r
$$

which together with Lemma 5.16 yields

$$
(k+1)^{n} \leq C \int_{0}^{\infty}\left|u^{\prime}\right|^{2} r^{n-1} d r .
$$

This completes the proof of Proposition 5.1.

\section{Proof of The UPPeR estimate}

The purpose of this section is to prove the following proposition and to complete the proof of Theorem 1.

Proposition 6.1. There exists a constant $C>0$ such that

$$
\int_{0}^{\infty}\left|u^{\prime}\right|^{2} r^{n-1} d r \leq C(k+1)^{n} \quad \text { for } u \in S_{k}
$$

We slightly modify the notation $E(r, \lambda)$, which has been defined for the initial data $\lambda$. However, from now on the energy is defined for any solution $u$ of (2.1) with (2.2).

Definition 6.2. We define $E(r, u)$ by

$$
E(r, u)=\frac{1}{2} u^{\prime}(r)^{2}+F(u(r)) \quad \text { for } \quad u \in S .
$$

Recall $E(r, u)>0$, which has been proved in Lemma 4.4. We consider the infimum of the energy at $r=1$.

Lemma 6.3. It holds that $\inf \{E(1, u): u \in S\}>0$.

Proof. Suppose that the assertion is false. Then there exists a sequence $\left\{u_{j}\right\} \subset$ $S$ such that $E\left(1, u_{j}\right)$ tends to 0 as $j \rightarrow \infty$. Therefore Lemma 4.14 implies that $\left\{u_{j}(0)\right\}$ is bounded. Set $\lambda_{j}=u_{j}(0)$. We choose a convergent subsequence (denoted again by $\lambda_{j}$ ) of $\left\{\lambda_{j}\right\}$ and denote its limit by $\lambda_{0}$. We set $u_{0}(r)=$ $u\left(r, \lambda_{0}\right)$. Then it follows that $E\left(1, u_{0}\right)=0$. The function $u_{0}(r)$ satisfies equation (2.1) but may not tend to 0 as $r \rightarrow \infty$, and hence $u_{0}(r)$ does not need to satisfy $E\left(r, u_{0}\right)>0$. On the other hand, since $\lambda_{j} \geq A_{*}$ or $\lambda_{j} \leq-B_{*}$ by Lemma 4.8 , it holds that $\lambda_{0} \neq-b, 0, a$. Therefore $E\left(r, u_{0}\right)$ is strictly decreasing, and in particular $E\left(2, u_{0}\right)<0$. The convergence of $\left\{u_{j}\right\}$ to $u_{0}$ in $C^{2}[0,2]$ implies that $E\left(2, u_{j}\right)<0$ for $j$ large enough. But this result contradicts the fact that $E(r, u)>0$ for $r \geq 0$ and $u \in S$. This contradiction completes the proof.

Definition 6.4. We define the constant $e_{0}$ by

$$
e_{0}=\inf \{E(1, u): u \in S\}>0 .
$$

We note that for each $u \in S$, the energy $E(r, u)$ is strictly decreasing and converges to 0 as $r \rightarrow \infty$. For $u \in S$ and $e \in\left(0, e_{0}\right)$, we denote by $T(u, e)$ the unique point $r$ which satisfies $E(r, u)=e$. From the definition of $e_{0}$, it follows that $T(u, e)>1$. 
The next proposition plays the most important role in the proof of Proposition 6.1.

Proposition 6.5. (i) If $e \in\left(0, e_{0}\right)$ is sufficiently small, then we have

$$
\int_{T}^{\infty} F(u) r^{n-1} d r<0 \text { for } T=T(u, e) \text { and } u \in S .
$$

(ii) For any $e>0$ sufficiently small, there exists a constant $C(e)>0$ depending only on $e$ such that

$$
\int_{T}^{\infty} u f(u) r^{n-1} d r+C(e) \int_{T}^{\infty} F(u) r^{n-1} d r<0 \text { for } T=T(u, e) \text { and } u \in S .
$$

This proposition will be proved later on. In what follows, we always fix $e$ so small that $0<e<\min \left(e_{0}, 1\right)$ and that the conclusion of Proposition 6.5 is valid. The application of Proposition 6.5 gives the next a priori estimate related to the integrals of $u^{\prime}(r)^{2}$ and $G(u)$.

Lemma 6.6. There is a constant $C>$ such that

$$
\int_{0}^{\infty}\left|u^{\prime}\right|^{2} r^{n-1} d r \leq C \int_{0}^{T} G(u) r^{n-1} d r \quad \text { for } T=T(u, e) \text { and } u \in S .
$$

Proof. We recall that $E(r) r^{n}$ tends to 0 as $r \rightarrow \infty$ by Lemma 4.4 (iv). Hence letting $R \rightarrow \infty$ in (4.18), we obtain

$$
(n-2) \int_{0}^{\infty}\left|u^{\prime}\right|^{2} r^{n-1} d r=2 n \int_{0}^{\infty} F(u) r^{n-1} d r
$$

We first deal with the case of $n=2$. Then the left-hand side of (6.2) vanishes, and so we have

$$
\int_{0}^{\infty} F(u) r d r=0
$$

or equivalently

$$
\int_{T}^{\infty} F(u) r d r=-\int_{0}^{T} F(u) r d r .
$$

We substitute this relation into Proposition 6.5 (ii) with $n=2$ to obtain

$$
\int_{T}^{\infty} u f(u) r d r \leq C(e) \int_{0}^{T} F(u) r d r \leq C(e) \int_{0}^{T} G(u) r d r,
$$

where we have used that $F(s)=G(s)-H(s) \leq G(s)$ for $s \in \mathbf{R}$ by Lemma 3.4 (ii).

We here prove that there is a constant $C_{1}>0$ such that

$$
s f(s) \leq C_{1} G(s) \text { for } s \in \mathbf{R} \text {. }
$$

Using (f5) and noting that $F(s)=G(s)-H(s) \leq G(s)$, we get a constant $C>0$ satisfying

$$
s f(s) \leq C F(s) \leq C G(s) \text { for }|s| \text { large enough. }
$$

Next, it follows from (f2) that

$$
s f(s) \leq 0 \leq G(s) \text { for } s \in[-b, a] .
$$


Hence the two inequalities above prove (6.4) since $G(s)>0$ for any $s \neq 0$. In view of (6.4) we have

$$
\int_{0}^{T} u f(u) r d r \leq C_{1} \int_{0}^{T} G(u) r d r .
$$

Summing up (6.3) and (6.5), we obtain

$$
\int_{0}^{\infty} u f(u) r d r \leq C \int_{0}^{T} G(u) r d r .
$$

We recall (5.33) with $n=2$, i.e.,

$$
\int_{0}^{\infty}\left|u^{\prime}\right|^{2} r d r=\int_{0}^{\infty} u f(u) r d r
$$

This together with (6.6) gives (6.1) in case of $n=2$.

We treat the case $n \geq 3$. Using (6.2) together with Proposition 6.5 (i), we have

$$
\int_{0}^{\infty}\left|u^{\prime}\right|^{2} r^{n-1} d r \leq \frac{2 n}{n-2} \int_{0}^{T} F(u) r^{n-1} d r \leq \frac{2 n}{n-2} \int_{0}^{T} G(u) r^{n-1} d r,
$$

where we have used that $F(s) \leq G(s)$ for $s \in \mathbf{R}$. The proof is now complete.

In view of the above lemma, we want to estimate the integral of $G(u) r^{n-1}$ over $[0, T]$ from above.

Lemma 6.7. There exists a constant $C>0$ such that

$$
\int_{0}^{T} G(u) r^{n-1} d r \leq C T^{n} \text { for } T=T(u, e) \text { and } u \in S .
$$

Proof. We substitute $R=T$ into (4.16) and use the inequality (4.19). Then it follows that

$$
2 E(T) T^{n}+(n-2) u^{\prime}(T) u(T) T^{n-1} \geq \nu \int_{0}^{T} F(u) r^{n-1} d r-\frac{C}{n} T^{n} .
$$

We see that $\left|u^{\prime}(T)\right|$ and $|u(T)|$ are bounded since $E(T)=e<1$. Therefore we have

$$
C T^{n}+C T^{n-1} \geq \nu \int_{0}^{T} F(u) r^{n-1} d r .
$$

We find a constant $C_{0}>0$ such that $F(s)=G(s)-H(s) \geq G(s)-C_{0}$ for $s \in \mathbf{R}$ because $H(s)$ is bounded on $\mathbf{R}$. Since $T \geq 1$, we have $T^{n} \geq T^{n-1}$, and so the above inequality yields (6.7). This completes the proof.

The inequality (6.7) provides a clue to establish a sharper estimate for the integral of $G(u) r^{n-1}$. In fact, from (6.7) we derive the following estimate.

Lemma 6.8. There exists a positive constant $C$ such that

$$
\left(\int_{0}^{T} G(u) r^{n-1} d r\right)^{1 / n} \leq C(k+1)+C \int_{t}^{T}(g(u) / u)^{1 / 2} d r
$$

for $t=t(u), T=T(u, e)$ and $u \in S_{k}$, where $t(u)$ is defined by Definition 5.13 .

Proof. We recall that $0<t<1<T$ by Lemma 4.12. Let $u \in S_{k}$. We set

$$
I=\{r \in(t, T):-b<u(r)<a\} .
$$


Since $F(u) \leq 0$ for $r \in I$, we have

$$
\frac{1}{2}\left|u^{\prime}\right|^{2} \geq \frac{1}{2}\left|u^{\prime}\right|^{2}+F(u)=E(r) \geq E(T)=e \quad \text { for } \quad r \in I,
$$

and therefore

$$
\left|u^{\prime}(r)\right| \geq \sqrt{2 e} \text { for } r \in I .
$$

Considering the outline of the graph of $u(r)$, we can write $I$ in the form of $I=\bigcup_{i=1}^{j}\left(\alpha_{i}, \beta_{i}\right)$ with som:e $j \leq k+1$. Here $\alpha_{i}$ and $\beta_{i}$ satisfy the following conditions :

(i) $u\left(\alpha_{i}\right)=a$ and $u\left(\beta_{i}\right)=-b$, or $u\left(\alpha_{i}\right)=-b$ and $u\left(\beta_{i}\right)=a$ for $1 \leq i \leq j-1$;

(ii) $u\left(\alpha_{j}\right)=a,-b$ and $u\left(\beta_{j}\right) \in[-b, a]$.

Fix $i \in[1, j]$ arbitrarily. By the mean-value theorem, there exists a point $\xi_{i} \in\left(\alpha_{i}, \beta_{i}\right)$ such that

$$
u\left(\beta_{i}\right)-u\left(\alpha_{i}\right)=u^{\prime}\left(\xi_{i}\right)\left(\beta_{i}-\alpha_{i}\right) .
$$

In any case of (i) and (ii) we have

$$
a+b \geq\left|u\left(\beta_{i}\right)-u\left(\alpha_{i}\right)\right|=\left|u^{\prime}\left(\xi_{i}\right)\right|\left(\beta_{i}-\alpha_{i}\right) .
$$

This together with $(6.8)$ yields

$$
\beta_{i}-\alpha_{i} \leq(a+b) / \sqrt{2 e} .
$$

Thus we estimate the measure of $I$ as

$$
|I|=\sum_{i=1}^{j}\left(\beta_{i}-\alpha_{i}\right) \leq C_{1}(k+1),
$$

where $C_{1}=(a+b) / \sqrt{2 e}$.

On the other hand, we set $I^{c}=[t, T] \backslash I$ to get

$$
\int_{t}^{T}(g(u) / u)^{1 / 2} d r \geq \int_{I^{c}}(g(u) / u)^{1 / 2} d r \geq \delta_{1}\left|I^{c}\right|
$$

where

$$
\delta_{1}=\min \left\{(g(s) / s)^{1 / 2}: s \in(-\infty,-b] \cup[a, \infty)\right\}>0 .
$$

Combining (6.9) and (6.10), we obtain

$$
\begin{aligned}
& C_{1}(k+1)+\delta_{1}^{-1} \int_{t}^{T}(g(u) / u)^{1 / 2} d r \\
& \quad \geq|I|+\left|I^{c}\right|=T-t \geq T-1 \geq C\left(\int_{0}^{T} G(u) r^{n-1} d r\right)^{1 / n}-1,
\end{aligned}
$$

where we have used Lemma 6.7 in the last inequality and employed $t(u) \leq 1$. The above inequality completes the proof.

Combining Lemmas 6.6, 6.8 and using several a priori estimates in Section 5 , we prove Proposition 6.1 in the following.

Proof of Proposition 6.1. Let $u \in S_{k}$. Integrating (5.17) over $[t, T]$ and using Lemma 5.5, we get

$$
(k+1) \pi \geq \varphi(T)-\varphi(t) \geq C_{2} \int_{t}^{T}(g(u) / u)^{1 / 2} d r+(n-1) \int_{t}^{T} \frac{u^{\prime} Y(u)}{r \rho^{2}} d r,
$$


which is rewritten as

$$
\int_{t}^{T}(g(u) / u)^{1 / 2} d r \leq C_{1}(k+1)+C_{2} \int_{t}^{T} \frac{\left|u^{\prime} Y(u)\right|}{r \rho^{2}} d r .
$$

For any $R>1$, we set

$$
R_{1}=\max (R, T) \text {. }
$$

Using Lemmas 5.14 and 5.15 and noting that $F(s) \leq G(s)$ for $s \in \mathbf{R}$, we have

$$
\begin{aligned}
\int_{t}^{R_{1}} \frac{\left|u^{\prime} Y(u)\right|}{r \rho^{2}} d r= & \left(\int_{t}^{R}+\int_{R}^{R_{1}}\right) \frac{\left|u^{\prime} Y(u)\right|}{r \rho^{2}} d r \\
\leq & C_{R, \varepsilon}\left(\int_{0}^{t} G(u) r^{n-1} d r\right)^{\varepsilon}+\frac{C_{\sigma}}{R} \int_{R}^{R_{1}}(g(u) / u)^{1 / 2} d r \\
& +C \sigma^{(\mu-2 q+1) / 2}(k+1),
\end{aligned}
$$

where $C_{R, \varepsilon}$ and $C_{\sigma}$ depend only on $R, \varepsilon$ and $\sigma$, respectively, but $C$ does not depend on anything. First, we set $\sigma=1$. Then (6.11) and (6.12) yield

$$
\begin{aligned}
\int_{t}^{T}(g(u) / u)^{1 / 2} d r \leq & C_{0}(k+1)+C_{R, \varepsilon}\left(\int_{0}^{t} G(u) r^{n-1} d r\right)^{\varepsilon} \\
& +\frac{C_{1}}{R} \int_{R}^{R_{1}}(g(u) / u)^{1 / 2} d r
\end{aligned}
$$

Secondly, we fix $R>1$ so large that $C_{1} / R<1 / 2$. For such $R$, if $R \geq T$, then $R_{1}=R$ and the last term in (6.13) vanishes. If $R<T$, then $R_{1}=T$, hence the last term is estimated from above by

$$
\frac{1}{2} \int_{t}^{T}(g(u) / u)^{1 / 2} d r \text {. }
$$

Then (6.13) yields

$$
\int_{t}^{T}(g(u) / u)^{1 / 2} d r \leq C(k+1)+C_{\varepsilon}\left(\int_{0}^{t} G(u) r^{n-1} d r\right)^{\varepsilon} .
$$

This together with Lemma 6.8 gives

$$
\left(\int_{0}^{T} G(u) r^{n-1} d r\right)^{1 / n} \leq C(k+1)+C_{\varepsilon}\left(\int_{0}^{t} G(u) r^{n-1} d r\right)^{\varepsilon}
$$

Lastly, noting that $t<1<T$ and setting $\varepsilon=1 / 2 n$, we obtain

$$
\left(\int_{0}^{T} G(u) r^{n-1} d r\right)^{1 / n} \leq C(k+1) \text {. }
$$

This inequality together with Lemma 6.6 yields

$$
\int_{0}^{\infty}\left|u^{\prime}\right|^{2} r^{n-1} d r \leq C(k+1)^{n}
$$

which proves Proposition 6.1.

To complete the proof of Proposition 6.1, we must prove Proposition 6.5. To this end, we introduce some notations. 
Definition 6.9. For $\delta>0, e>0, u \in S$ and $T=T(u, e)$, we set

$$
\begin{aligned}
& I^{+}=\{r \in(T, \infty): 0 \leq u(r)<a\}, \\
& I^{-}=\{r \in(T, \infty):-b<u(r)<0\}, \\
& J^{+}=\{r \in(T, \infty): u(r)>A-\delta\}, \\
& J^{-}=\{r \in(T, \infty): u(r)<-B+\delta\} .
\end{aligned}
$$

Since $T$ depends on $u$ and $e$, the sets $I^{+}$and $I^{-}$depend only on $u$ and $e$, however, $J^{+}$and $J^{-}$depend on $u, e$ and $\delta$ as well. The proof of Proposition 6.5 is based on the next lemma.

Lemma 6.10. For any $e>0$ sufficiently small there exists $a \delta>0$ such that

$$
\begin{aligned}
& \int_{J^{+}} u f(u) r^{n-1} d r \leq C_{0} \sqrt{e} \int_{I^{+}}|u f(u)| r^{n-1} d r \quad \text { for } \quad u \in S, \\
& \int_{J^{-}} u f(u) r^{n-1} d r \leq C_{0} \sqrt{e} \int_{I^{-}}|u f(u)| r^{n-1} d r \quad \text { for } \quad u \in S,
\end{aligned}
$$

where $C_{0}$ is a positive constant independent of $u, e$ and $\delta$.

Proof. We prove assertion (i) only since assertion (ii) can be proved in the same way. Let $u \in S$. In the case where $J^{+}=\varnothing$, the assertion is trivial. Let $J^{+} \neq \varnothing$. Then $I^{+}$is also nonempty since $u(r)$ tends to 0 as $r \rightarrow \infty$. Let $0<e<\min \left(1, e_{0}\right)$. Then we have

$$
\frac{1}{2} u^{\prime}(r)^{2}+F(u(r))=E(r) \leq E(T)=e<1 \text { for } r \geq T .
$$

Note that $F(s)$ has local minima at $s=-b, a$, and we set

$$
-F_{0}=\min \{F(-b), F(a)\}=\inf \{F(s): s \in \mathbf{R}\}<0 .
$$

Then (6.14) implies that

$$
\left|u^{\prime}(r)\right| \leq C_{1} \equiv \sqrt{2\left(1+F_{0}\right)} \quad \text { for } \quad r \geq T .
$$

On the other hand, since $F(s)$ is increasing in $(a, \infty)$, it holds that $F(u(r)) \geq$ $F(A-\delta)$ for $r \in J^{+}$. Hence (6.14) implies that

$$
\left|u^{\prime}(r)\right|^{2} \leq 2 e-2 F(A-\delta) \text { for } r \in J^{+} .
$$

Since $F(A)=0$, we can choose $\delta>0$ so small that

$$
|F(A-\delta)| \leq e \text { and }(A+a) / 2<A-\delta<A .
$$

Then (6.16) gives

$$
\left|u^{\prime}(r)\right| \leq 2 \sqrt{e} \text { for } r \in J^{+} .
$$

We here observe (6.14) again, which gives directly

$$
F(u(r))<1 \text { for } r \geq T .
$$

Hence we set $\bar{A}=\sup \{s: F(s) \leq 1\}$ to obtain

$$
(A+a) / 2<A-\delta \leq u(r) \leq \bar{A} \text { for } r \in J^{+} .
$$

Since $f(s)>0$ for $s>a$ by (f2), we set

$$
m=\min \{f(s):(A+a) / 2 \leq s \leq \bar{A}\}>0 .
$$


Using (6.18) and noting that $r \geq T \geq 1$, we obtain

$$
-u^{\prime \prime}=f(u)+\frac{n-1}{r} u^{\prime} \geq m-2(n-1) \sqrt{e} \geq \frac{m}{2} \text { for } r \in J^{+},
$$

if $e>0$ is so small that

$$
\sqrt{e} \leq \frac{m}{4(n-1)} .
$$

In view of Lemma 4.6, we can write $J^{+}$as $J^{+}=\bigcup_{i=1}^{j}\left(\alpha_{i}, \beta_{i}\right)$, where $u\left(\alpha_{i}\right)=$ $u\left(\beta_{i}\right)=A-\delta$ for $2 \leq i \leq j, u\left(\alpha_{1}\right) \geq A-\delta, u\left(\beta_{1}\right)=A-\delta$. Integrating (6.20) over $\left[\alpha_{i}, \beta_{i}\right]$ and using $(6.18)$, we get

$$
4 \sqrt{e} \geq-u^{\prime}\left(\beta_{i}\right)+u^{\prime}\left(\alpha_{i}\right) \geq \frac{m}{2}\left(\beta_{i}-\alpha_{i}\right),
$$

or equivalently

$$
0<\beta_{i}-\alpha_{i} \leq \frac{8}{m} \sqrt{e}
$$

In view of (6.19), we set $M=\max \{s f(s):(A+a) / 2 \leq s \leq \bar{A}\}$ to obtain

$$
\int_{\alpha_{i}}^{\beta_{i}} u f(u) r^{n-1} d r \leq M \beta_{i}^{n-1}\left(\beta_{i}-\alpha_{i}\right) \leq \frac{8 M}{m} \sqrt{e} \beta_{i}^{n-1} .
$$

Let us estimate the integral of $|u f(u)|$ over $I^{+}$. To do so, we define a subinterval $\left(\gamma_{i}, \delta_{i}\right)$ of $I^{+}$. For $1 \leq i \leq j$, we set

$$
\begin{aligned}
& \gamma_{i}=\min \left\{r \in\left[\beta_{i}, \infty\right): u(r)=a / 2\right\}, \\
& \delta_{i}=\min \left\{r \in\left[\gamma_{i}, \infty\right): u(r)=a / 4\right\} .
\end{aligned}
$$

Therefore it holds that

$$
\begin{gathered}
\alpha_{1}<\beta_{1}<\gamma_{1}<\delta_{1}<\alpha_{2}<\cdots<\delta_{j}, \\
a / 4<u(r)<a / 2, \quad u^{\prime}(r)<0 \text { for } r \in\left(\gamma_{i}, \delta_{i}\right) .
\end{gathered}
$$

Using (6.15), we have

$$
-u^{\prime}(r) \leq C_{1} \text { for } r \in\left(\gamma_{i}, \delta_{i}\right) .
$$

Integrating both sides over $\left[\gamma_{i}, \delta_{i}\right]$, we obtain

$$
\frac{a}{4}=-u\left(\delta_{i}\right)+u\left(\gamma_{i}\right) \leq C_{1}\left(\delta_{i}-\gamma_{i}\right)
$$

that is,

$$
C_{2} \equiv \frac{a}{4 C_{1}} \leq \delta_{i}-\gamma_{i}
$$

We set $m_{1}=\min \{|s f(s)|: a / 4 \leq s \leq a / 2\}>0$. Then (6.24) yields

$$
\int_{\gamma_{i}}^{\delta_{i}}|u f(u)| r^{n-1} d r \geq m_{1} \gamma_{i}^{n-1}\left(\delta_{i}-\gamma_{i}\right) \geq m_{1} C_{2} \beta_{i}^{n-1}
$$

Combining (6.23) and (6.25), we obtain

$$
\int_{\alpha_{i}}^{\beta_{i}} u f(u) r^{n-1} d r \leq C_{3} \sqrt{e} \int_{y_{i}}^{\delta_{i}}|u f(u)| r^{n-1} d r,
$$


where $C_{3}=8 \mathrm{M} / \mathrm{mm}_{1} C_{2}$. We note that $\left(\gamma_{i}, \delta_{i}\right) \subset I^{+}$. Summing up both sides on $i$, we have

$$
\int_{J^{+}} u f(u) r^{n-1} d r \leq C_{3} \sqrt{e} \int_{I^{+}}|u f(u)| r^{n-1} d r .
$$

Note that $C_{3}$ is independent of $u, e$ and $\delta$. In view of (6.21), we set

$$
e_{1}=\min \left\{1, e_{0},(m / 4(n-1))^{2}\right\} .
$$

For given $e \in\left(0, e_{1}\right)$ we choose a small $\delta>0$ which satisfies (6.17). Then the choice of $\delta$ yields (6.26), which is the desired inequality (i).

To prove Proposition 6.5, we prepare the next notations and need another lemma.

Definition 6.11. For $u \in S$ we set

$$
\begin{aligned}
K^{+} & =\{r \in(T, \infty): 0 \leq u(r)<A\}, \\
K^{-} & =\{r \in(T, \infty):-B<u(r)<0\}, \\
L^{+} & =\{r \in(T, \infty): u(r) \geq A\}, \\
L^{-} & =\{r \in(T, \infty): u(r) \leq-B\} .
\end{aligned}
$$

Lemma 6.12. If $e>0$ is sufficiently small, then for any $u \in S$ we have

$$
\begin{aligned}
& \int_{L^{+}} F(u) r^{n-1} d r \leq \frac{1}{2} \int_{K^{+}}|F(u)| r^{n-1} d r=-\frac{1}{2} \int_{K^{+}} F(u) r^{n-1} d r, \\
& \int_{L^{-}} F(u) r^{n-1} d r \leq \frac{1}{2} \int_{K^{-}}|F(u)| r^{n-1} d r=-\frac{1}{2} \int_{K^{-}} F(u) r^{n-1} d r .
\end{aligned}
$$

Proof. We prove assertion (i) only, however, (ii) can be treated in the same way. By assumptions (f2) and (f5), there exists a constant $C_{1}>0$ such that

$$
F(s) \leq C_{1} s f(s) \text { for } s \geq A .
$$

Next, we note that $F(s) \neq 0$ for $0<s \leq a$ and $F(0)=0$. Then there exists a positive constant $C_{2}$ by (f3) such that

$$
|s f(s)| \leq C_{2}|F(s)| \text { for } 0 \leq s \leq a .
$$

Compare Definitions 6.9 and 6.11. Then we see that $L^{+} \subset J^{+}$and $I^{+} \subset K^{+}$. Hence Lemma $6.10,(6.27)$ and (6.28) imply that

$$
\begin{aligned}
\int_{L^{+}} F(u) r^{n-1} d r & \leq C_{1} \int_{L^{+}} u f(u) r^{n-1} d r \leq C_{1} \int_{J^{+}} u f(u) r^{n-1} d r \\
& \leq C_{0} C_{1} \sqrt{e} \int_{I^{+}}|u f(u)| r^{n-1} d r \leq C_{0} C_{1} C_{2} \sqrt{e} \int_{I^{+}}|F(u)| r^{n-1} d r \\
& \leq C_{0} C_{1} C_{2} \sqrt{e} \int_{K^{+}}|F(u)| r^{n-1} d r .
\end{aligned}
$$

We choose $e>0$ so small that $C_{0} C_{1} C_{2} \sqrt{e}<1 / 2$. This completes the proof.

We are now ready for proving Proposition 6.5.

Proof of Proposition 6.5. Summing up (i) and (ii) in Lemma 6.12, we have

$$
\int_{T}^{\infty} F(u) r^{n-1} d r \leq \frac{1}{2} \int_{K} F(u) r^{n-1} d r,
$$


where

$$
K=K^{+} \cup K^{-}=\{r \in(T, \infty):-B<u(r)<A\} .
$$

We note that $F(u(r))<0$ for $r \in K$. Hence the inequality (6.29) proves assertion (i) of Proposition 6.5.

We show assertion (ii). For small $\delta>0, e>0$ and any $u \in S$, we define

$$
\begin{aligned}
W & =W^{+} \cup W^{-}, \\
W^{+} & =\{r \in(T, \infty): a \leq u(r) \leq A-\delta\}, \\
W^{-} & =\{r \in(T, \infty):-B+\delta \leq u(r) \leq-b\} .
\end{aligned}
$$

We choose $e>0$ so small that $C_{0} \sqrt{e}<1$, and then $\delta>0$ is determined by Lemma 6.10. Since $u f(u) \leq 0$ for $r \in I^{+} \cup I^{-}$, the inequalities (i) and (ii) in Lemma 6.10 are rewritten as

$$
\begin{aligned}
& \int_{J^{+}} u f(u) r^{n-1} d r \leq-\int_{I^{+}} u f(u) r^{n-1} d r, \\
& \int_{J^{-}} u f(u) r^{n-1} d r \leq-\int_{I^{-}} u f(u) r^{n-1} d r .
\end{aligned}
$$

Note that $I^{+} \cup I^{-} \cup J^{+} \cup J^{-}=(T, \infty) \backslash W$. Then we sum up the two inequalities above to obtain

$$
\int_{T}^{\infty} u f(u) r^{n-1} d r \leq \int_{W} u f(u) r^{n-1} d r
$$

On the other hand, since $F(s)<0$ for $s \in(-B, A) \backslash\{0\}$ and $K \supset W$, the inequality (6.29) yields

$$
\int_{T}^{\infty} F(u) r^{n-1} d r \leq-\frac{1}{2} \int_{K}|F(u)| r^{n-1} d r \leq-\frac{1}{2} \int_{W}|F(u)| r^{n-1} d r .
$$

Summing up (6.30) and the above inequality multiplied by $C>0$, we obtain

$$
\int_{T}^{\infty} u f(u) r^{n-1} d r+C \int_{T}^{\infty} F(u) r^{n-1} d r \leq \int_{W}\left(u f(u)-\frac{C}{2}|F(u)|\right) r^{n-1} d r
$$

for any $C>0$. We set

$$
\begin{gathered}
M=\max \{s f(s): s \in[-B+\delta,-b] \cup[a, A-\delta]\}>0, \\
m=\min \{|F(s)|: s \in[-B+\delta,-b] \cup[a, A-\delta]\}>0 .
\end{gathered}
$$

We now choose $C>0$ so large that $M-m C / 2<0$. Then the right-hand side of (6.31) is negative. This proves Proposition 6.5(ii) and the proof is complete.

We conclude this paper by proving Theorem 1 .

Proof of Theorem 1. By Propositions 5.1 and 6.1, there exist constants $C_{1}, C_{2}$ $>0$ such that

$$
C_{1}(k+1)^{n} \leq \int_{0}^{\infty}\left|u^{\prime}\right|^{2} r^{n-1} d r \leq C_{2}(k+1)^{n}
$$

for $u \in S_{k}$ and $k \geq 0$. We estimate the Lagrangian $L(u)$ defined by (1.9), i.e.,

$$
L(u)=\int_{\mathbf{R}^{n}}\left(\frac{1}{2}|\nabla u|^{2}-F(u)\right) d x=\omega \int_{0}^{\infty}\left(\frac{1}{2}\left|u^{\prime}\right|^{2}-F(u)\right) r^{n-1} d r,
$$


where $\omega$ means the surface area of the unit sphere $\left\{x \in \mathbf{R}^{n}:|x|=1\right\}$. We show that

$$
L(u)=\frac{\omega}{n} \int_{0}^{\infty}\left|u^{\prime}\right|^{2} r^{n-1} d r \quad \text { for } \quad u \in S .
$$

To this end, we recall the identities (5.33) and (6.2), i.e.,

$$
\begin{gathered}
\int_{0}^{\infty}\left|u^{\prime}\right|^{2} r^{n-1} d r=\int_{0}^{\infty} u f(u) r^{n-1} d r, \\
(n-2) \int_{0}^{\infty}\left|u^{\prime}\right|^{2} r^{n-1} d r=2 n \int_{0}^{\infty} F(u) r^{n-1} d r .
\end{gathered}
$$

The substitution of (6.36) into (6.33) gives (6.34). By (6.32) and (6.34), we obtain the estimate

$$
C_{3}(k+1)^{n} \leq L(u) \leq C_{4}(k+1)^{n} \quad \text { for } \quad u \in S_{k} .
$$

We now consider the special cases where $f(u)=|u|^{p-1} u-u$ and $f(u)=$ $u \log |u|$. First, we treat $f(u)=|u|^{p-1} u-u$. Then $F(u)=|u|^{p+1} /(p+1)-u^{2} / 2$. Therefore the inequalities (6.35) and (6.36) become

$$
\begin{gathered}
\int_{0}^{\infty}\left|u^{\prime}\right|^{2} r^{n-1} d r=\int_{0}^{\infty}|u|^{p+1} r^{n-1} d r-\int_{0}^{\infty} u^{2} r^{n-1} d r, \\
(n-2) \int_{0}^{\infty}\left|u^{\prime}\right|^{2} r^{n-1} d r=\frac{2 n}{p+1} \int_{0}^{\infty}|u|^{p+1} r^{n-1} d r-n \int_{0}^{\infty} u^{2} r^{n-1} d r .
\end{gathered}
$$

These identities imply

$$
\int_{0}^{\infty}\left|u^{\prime}\right|^{2} r^{n-1} d r=\frac{n(p-1)}{2(p+1)} \int_{0}^{\infty}|u|^{p+1} r^{n-1} d r=\frac{n(p-1)}{n+2-(n-2) p} \int_{0}^{\infty} u^{2} r^{n-1} d r .
$$

Each coefficient of the integrals is positive. This relation and (6.32) give

$$
C_{5}(k+1)^{n / 2} \leq\|u\|_{L^{2}} \leq\|u\|_{H^{1}} \leq C_{6}(k+1)^{n / 2} .
$$

Let $f(u)=u \log |u|$. Then $F(u)=(1 / 2) u^{2} \log |u|-(1 / 4) u^{2}$. Substituting these functions into (6.35) and (6.36), we obtain

$$
\int_{0}^{\infty}\left|u^{\prime}\right|^{2} r^{n-1} d r=\int_{0}^{\infty} u^{2} \log |u| r^{n-1} d r=\frac{n}{4} \int_{0}^{\infty} u^{2} r^{n-1} d r
$$

This identity together with $(6.32)$ yields $(6.37)$. The proof is thereby complete.

\section{REFERENCES}

1. H. Berestycki and P.-L. Lions, Nonlinear scalar field equations, II. Existence of infinitely many solutions, Arch. Rational Mech. Anal. 82 (1983), 347-375.

2. M. Grillakis, Existence of nodal solutions of semilinear equations in $\mathbf{R}^{n}$, J. Differential Equations 85 (1990), 367-400.

3. P. Hartman, Ordinary differential equations, 2nd ed., Birkhäuser, Boston, 1982.

4. C. Jones and T. Küpper, On the infinitely many solutions of a semilinear elliptic equation, SIAM J. Math. Anal. 17 (1986), 803-835.

5. R. Kajikiya, Sobolev norms of radially symmetric oscillatory solutions for superlinear elliptic equations, Hiroshima Math. J. 20 (1990), 259-276. 
6. __ Radially symmetric solutions of semilinear elliptic equations, existence and Sobolev estimates, Hiroshima Math. J. 21 (1991), 111-161.

7. $\ldots$, Nodal solutions of superlinear elliptic equations in symmetric domains, Advances in Math. Sci. Appl. 3 (1994), 219-266.

8. K. McLeod, W. C. Troy and F. B. Weissler, Radial solutions of $\Delta u+f(u)=0$ with prescribed numbers of zeros, J. Differential Equations 83 (1990), 368-378.

9. S. I. Pohozaev, Eigenfunctions of the equation $\Delta u+\lambda f(u)=0$, Soviet Math. Dokl. 5 (1965), 1408-1411.

10. W. A. Strauss, Existence of solitary waves in higher dimensions, Comm. Math. Phys. 55 (1977), 149-162.

Department of Applied Mathematics, Faculty of Engineering, Miyazaki University, Kibana, MiYazaKi 889-21, JaPAN

E-mail address: t06209u@cc.miyazaki-u.ac.jp 